\title{
Whose opinion counts? Political processes and the implementation problem
}

Citation for published version (APA):

Saran, R. R. S., \& Tumennasan, N. (2011). Whose opinion counts? Political processes and the implementation problem. METEOR, Maastricht University School of Business and Economics. METEOR Research Memorandum No. 019 https://doi.org/10.26481/umamet.2011019

Document status and date:

Published: 01/01/2011

DOI:

10.26481/umamet.2011019

Document Version:

Publisher's PDF, also known as Version of record

\section{Please check the document version of this publication:}

- A submitted manuscript is the version of the article upon submission and before peer-review. There can be important differences between the submitted version and the official published version of record.

People interested in the research are advised to contact the author for the final version of the publication, or visit the DOI to the publisher's website.

- The final author version and the galley proof are versions of the publication after peer review.

- The final published version features the final layout of the paper including the volume, issue and page numbers.

Link to publication

\footnotetext{
General rights rights.

- You may freely distribute the URL identifying the publication in the public portal. please follow below link for the End User Agreement:

www.umlib.nl/taverne-license

Take down policy

If you believe that this document breaches copyright please contact us at:

repository@maastrichtuniversity.nl

providing details and we will investigate your claim.
}

Copyright and moral rights for the publications made accessible in the public portal are retained by the authors and/or other copyright owners and it is a condition of accessing publications that users recognise and abide by the legal requirements associated with these

- Users may download and print one copy of any publication from the public portal for the purpose of private study or research.

- You may not further distribute the material or use it for any profit-making activity or commercial gain

If the publication is distributed under the terms of Article $25 \mathrm{fa}$ of the Dutch Copyright Act, indicated by the "Taverne" license above, 


\section{Maastricht University}

Rene Saran, Norovsambuu

Tumennasan

Whose Opinion Counts? Political

Processes and the

I mplementation Problem

RM/11/019

\section{METEOR}

Maastricht University School of Business and Economics

Maastricht Research School of Economics

of Technology and Organization

P.O. Box 616

NL - 6200 MD Maastricht

The Netherlands 


\title{
Whose Opinion Counts?
}

\section{Political Processes and the Implementation Problem*}

\author{
Rene $\operatorname{Saran}^{\dagger} \quad$ Norovsambuu Tumennasan ${ }^{\ddagger}$
}

February 14, 2011

\begin{abstract}
The mechanism used in Nash implementation is a form of direct democracy, taking everyone's opinion into account. We augment this mechanism with a political process that selects the opinions of a subset of the individuals. We study three such processes - oligarchy, oligarchic democracy and random sampling - and compare the social choice rules (SCRs) that can be implemented using each of these processes with those that can be Nash implemented. In oligarchy, only the opinions of a fixed subset of the individuals - the oligarchs - determine the implemented alternative. We obtain a negative result for oligarchies: there exist Nash implementable SCRs that cannot be implemented by any oligarchy. Oligarchic democracy is a perturbation of oligarchy, in which the opinions of the oligarchs "almost always" determine the implemented alternative but sometimes, everyone's opinions are considered. In a sharp contrast to the negative result for oligarchies, we show that in economic environments, every Nash implementable SCR can be implemented by an oligarchic democracy in which any three individuals act as oligarchs. In random sampling, opinions of a fixed number of individuals are selected randomly, which then determine the implemented alternative. We show that in economic environments, every Nash implementable SCR can be implemented by randomly sampling opinions of four individuals.
\end{abstract}

Keywords: Nash Implementation; Direct Democracy; Oligarchy; Oligarchic Democracy; Random Sampling

JEL: C72; D78

*We thank Bo Chen, Alexander Koch, David Levine, Rudolf Müller, Julia Nafziger, Ronald Peeters, William Sandholm, Ricardo Serrano-Padial, William Thomson and seminar audience at Aarhus and Copenhagen for useful comments and suggestions.

${ }^{\dagger}$ Department of Economics, Maastricht University, PO Box 616, 6200 MD Maastricht, The Netherlands. Email: r.saran@maastrichtuniversity.nl; Tel: +31-43-3883763; Fax: +31-43-3884878

${ }^{\ddagger}$ School of Economics and Management, Aarhus University, Bartholins Allé 10, Aarhus C 8000, Denmark. Email: ntumennasan@econ.au.dk; Tel: +45 89421510 


\section{Introduction}

Consider a community of $n$ individuals having a social choice rule (SCR) that specifies the socially desirable alternatives conditional on the true preferences of these individuals. This community would like to design a mechanism such that individuals' incentives in the mechanism are "aligned" with its SCR, i.e., given their true preferences, any Nash equilibrium of the mechanism must lead to a socially desirable alternative and every socially desirable alternative must be obtained under some Nash equilibrium of the mechanism. This is the implementation problem under complete information. ${ }^{1}$ The standard mechanism in this literature is defined by a set of messages for each individual and an outcome function mapping each message profile to an alternative. This mechanism is a form of direct democracy since every individual's message is considered in the outcome function. However, direct democracy is seldom used by communities to make decisions at national, state or even local levels. Indeed, when $n$ is large, it will be too costly and time consuming to collect opinions of every individual in the community while making social decisions. By the time a data collector gathers everyone's opinions, the state of the economy might change, rendering the whole exercise meaningless. In this paper, we augment the standard mechanism with a political process that selects a subset of the individuals, which we call a senate. The messages of the selected senators determine the implemented alternative while the messages of all other individuals are ignored. We are interested in the relationship between a political process and the SCRs that a community can implement if it were to adopt that political process.

In his seminal contribution, Maskin (1999) studied Nash implementation which, using our terminology, is the implementation problem under direct democracy. He showed that any Nash implementable SCR must be Maskin monotonoic. Maskin (1999) further proved that if $n \geq 3$, then Maskin monotonicity and no-veto power are sufficient conditions for a SCR to be Nash implementable. ${ }^{2}$ It follows that in economic environments (i.e., when at most $n-2$ individuals have a common most-preferred alternative) with at least 3 individuals, Maskin monotonicity is sufficient for Nash implementation of a SCR. We use these results for direct democracy as benchmarks. In particular, for each political process studied in this paper, we are interested in knowing whether every SCR that is Nash implementable is also implementable using that political process. If the answer is yes, then replacing direct democracy with such a political process based on messages of only a small number of individuals can reduce the costs and time involved in collecting everyone's opinions. ${ }^{3}$

\footnotetext{
${ }^{1}$ See Maskin and Sjöström (2002) and Serrano (2004) for surveys of implementation theory.

${ }^{2}$ For necessary and sufficient conditions for Nash implementation see Moore and Repullo (1990), Sjöström (1991), Danilov (1992) and Yamato (1992).

${ }^{3}$ We do not explicitly model such costs of collecting messages. One possibility is to assume that there
} 
We restrict attention to three political processes: oligarchy, oligarchic democracy and random sampling. In an oligarchy, the senate is exogenously fixed to a strict subset of the individuals, whom we refer to as the oligarchs. Thus, only the messages of the oligarchs determine the implemented alternative in an oligarchy. Oligarchic democracy is a perturbation of the oligarchy in which the senate is "almost always" the exogenously fixed set of oligarchs but there is a small chance of "regulating" this oligarchy through direct democracy. That is, in an oligarchic democracy, the messages of only the oligarchs determine the implemented alternative with probability "arbitrarily" close to 1 while with the rest of the probability, the messages of all individuals determine the implemented alternative. Finally, in random sampling, an exogenously fixed number of individuals, which is strictly less than $n$, are selected at random to form the senate and only the messages of these selected individuals determine the implemented alternative. ${ }^{4}$

A mechanism is now defined by a set of messages for each player, a political process that selects the senate, and a set of outcome functions, one for each possible senate, mapping messages of the senators to alternatives. This mechanism defines a strategic-form game in which all individuals simultaneously announce their messages. The political process then selects the senate and finally, the messages of the selected senators determine the implemented alternative. Since the selection of the senate is stochastic, for any given message profile, the outcome could be a lottery over the set of alternatives. We study the SCRs that are implementable in Nash equilibrium of this game. That is, we require that every Nash equilibrium outcome of the game is a socially desirable alternative and every socially desirable alternative must be obtained as a Nash equilibrium outcome of the game. Thus, Nash equilibrium outcomes are deterministic (i.e., the same alternative is implemented for all possible senates) and hence, only off-the-equilibrium outcomes can be lotteries.

We must point out that it is possible to alter the mechanisms under each political process so that an individual announces a message if and only if she is selected as the senator. There is no change in our results under this participation requirement, which seems more reasonable

is a fixed cost of collecting each message equal to $c$. In Section 6 , we briefly discuss the implication of our results under this assumption. We must also point out that we do not consider the issue of communication and processing burdens imposed on, respectively, the individuals and mechanism designer due to large size of the message space (see, for e.g., Saijo (1988) and McKelvey (1989), who tackle this issue in the context of Nash implementation).

${ }^{4}$ The selection of the senate in all these political processes is exogenous. Alternatively, one can consider political processes in which the senate is selected endogenously, e.g., through elections. This alternative model will be appropriate to situations when the community is aware of the implementation problem at the time of the elections. On the other hand, if a new implementation problem arises after the elections and the elected senate has a fixed term (e.g., due to an unanticipated financial crisis, the current senate must vote on bailouts for financial institutions), then the model of oligarchy might be more appropriate. Nevertheless, endogenous selection of senators offers an interesting area of research that we hope will be pursued in future. 
in socio-economic problems. A description of our results follows.

We show that any SCR that is implementable by oligarchy is also Nash implementable by making the outcome function under the direct democracy unresponsive to the messages of the commoners (individuals who are not in the oligarchy). However, the converse is not true: we give an example of a SCR that is Nash implementable but it is not implementable under any oligarchy. The reason is that the necessary condition for implementation under oligarchy is stronger than Maskin monotonicity. This necessary condition says that if some alternative $a$ drops out of the SCR when the preferences change, then for some oligarch, there must be a reversal of her preferences over alternatives around $a$ (i.e., there exists alternative $b$ which goes from being weakly worse than $a$ to strictly preferred to $a$ ). In contrast, Maskin monotonicity requires the same preference reversal for some individual.

The perturbed process of oligarchic democracy, on the other hand, generates dramatically opposite conclusions. We show that in economic environments, any Nash implementable SCR is implementable by any oligarchic democracy that has at least three oligarchs. The identity of the oligarchs does not matter for this result; any three players can be designated as the oligarchs. In fact, the necessary and sufficient condition for implementation by oligarchic democracy is even weaker than Maskin monotonicity. ${ }^{5}$ Thus, "regulation" of the oligarchy by a "minimal" probability of direct democracy makes it possible to implement a larger class of SCRs in economic environments. This success of oligarchic democracy, however, does not carry over to non-economic environments. We give an example of a non-economic environment and a SCR that is Nash implementable but not implementable by oligarchic democracy with any subset of the individuals designated as oligarchs. The reason for this failure of oligarchic democracy is the lack of "sufficient" diversity in the sets of most-preferred alternatives of the oligarchs. As we show, any SCR satisfying Maskin monotonicity and noveto power can be implemented by an oligarchic democracy of at least three oligarchs who never have a common most-preferred alternative. Furthermore, whenever such oligarchs exist and everyone has a unique most-preferred alternative (e.g., strict preferences, single-peaked preferences), then any Maskin monotonic SCR can be implemented by oligarchic democracy; thus, we do not need the SCR to satisfy no-veto power in this case.

The last political process that we study is random sampling. We show that in economic environments, any Nash implementable SCR is implementable by random sampling of at least four individuals (actually, we prove this for a slightly larger class of environments). Thus, in economic environments, instead of collecting the messages of everyone in the community,

\footnotetext{
${ }^{5}$ This is because the oligarchs can generate lotteries by changing their messages and we use the information about their preferences over lotteries to construct the mechanism. Benoit and Ok (2008, Remark 3) provide an example to show that Maskin monotonicity is not necessary for Nash implementation if the planner can implement lotteries and use the information about individuals' preferences over lotteries.
} 
we simply have to collect the messages of four randomly selected individuals in order to implement any Nash implementable SCR. It turns out, four is in fact the minimal sample size that guarantees this result. We give an example of a SCR that is Nash implementable in an economic environment but it is not implementable by random sampling when the sample size is smaller than four.

Bochet (2007) and Benoît and Ok (2008) introduce lottery mechanisms in the implementation problem under complete information. In a lottery mechanism, lotteries over alternatives can be implemented at off-the-equilibrium messages. Thus, like in our notion of implementation, equilibrium outcomes are deterministic. Under mild domain restrictions, these authors show that Maskin monotonicity is both necessary and sufficient for implementation of a SCR using lottery mechanisms. ${ }^{6}$ In general, an off-the-equilibrium message profile can be mapped to any arbitrary lottery in a lottery mechanism. On the other hand, in our oligarchic democracy and random sampling processes, the "structure" of the lottery is constrained by the particular stochastic selection of the senate since once a senate has been selected, a pure alternative is implemented. Nevertheless, in their proofs, Bochet (2007) and Benoît and Ok (2008) use simple lotteries with at most two alternatives in their respective supports. This is similar to the lottery generated under oligarchic democracy (because the senate is either the oligarchs or all individuals). In contrast to Bochet (2007) and Benoît and Ok (2008), however, commoners cannot induce a lottery in oligarchic democracy since their messages are considered only in one senate.

The rest of the paper is organized as follows. We outline the implementation problem and provide preliminary definitions in Section 2. In Sections 3, 4 and 5, we present the results for, respectively, implementation by oligarchy, oligarchic democracy and random sampling. We provide a brief conclusion in Section 6. Longer proofs are collected in Appendix.

\section{Preliminaries}

There is a finite set of players $N=\{1, \ldots, n\}$ with $n \geq 3$. The set of social alternatives is $A$, which can be infinite but not singleton. A lottery $l$ is a probability distribution with a finite support in $A$. For any lottery $l$, let $l(a)$ denote the probability assigned by $l$ to alternative $a \in A$. We write $a$ for both the alternative $a \in A$ and the degenerate lottery that puts probability 1 on the alternative $a$. Let $\Delta A$ denotes the set of lotteries.

Let $\Theta$ be set of states with at least two elements. A typical state is denoted by $\theta$. We assume that the players have complete information about the realized state.

\footnotetext{
${ }^{6}$ To prove the necessary part, they constrain the planner to use only the information about individuals' preferences over alternatives.
} 
Each player has a state dependent preference ordering (i.e., complete and transitive relation) $\succeq_{i}^{\theta}$ over $\Delta A .^{7}$ Let $\succ_{i}^{\theta}$ and $\sim_{i}^{\theta}$ denote, respectively, the strict and indifference relations derived from $\succeq_{i}^{\theta}$.

For any $i, \theta$ and for all natural numbers $k \geq 1$, define:

$$
\begin{aligned}
P_{1}(i, \theta) & =\left\{a \in A: a \succeq_{i}^{\theta} a^{\prime}, \forall a^{\prime} \in A\right\} \\
P_{k+1}(i, \theta) & =\left\{a \in A \backslash P_{k}(i, \theta): a \succeq_{i}^{\theta} a^{\prime}, \forall a^{\prime} \in A \backslash P_{k}(i, \theta)\right\}
\end{aligned}
$$

Thus, $P_{1}(i, \theta)$ is the set of most-preferred alternatives for player $i$ in state $\theta, P_{2}(i, \theta)$ is the set of second preferred alternatives for player $i$ in state $\theta$ and so on.

Assumption 2.1. Preferences over Lotteries are Monotone: We assume that for all $i \in N$, $\theta \in \Theta$, and $l, l^{\prime} \in \Delta A$, we have

$$
\sum_{k=1}^{K} \sum_{a \in P_{k}(i, \theta)} l(a) \geq \sum_{k=1}^{K} \sum_{a \in P_{k}(i, \theta)} l^{\prime}(a), \forall K \geq 1 \Longrightarrow l \succeq_{i}^{\theta} l^{\prime}
$$

and whenever at least one inequality is strict, then $l \succ_{i}^{\theta} l^{\prime}$.

Hence, shifting probability from alternatives lower in the preference ordering to alternatives higher in the preference ordering generates a preferred lottery.

\section{$2.1 \quad$ Environment}

The environment is $\mathcal{E}=\left\langle N, A,\left(\left(\succeq_{i}^{\theta}\right)_{i \in N}\right)_{\theta \in \Theta}\right\rangle$. We consider different classes of environments, the most important being economic environment as defined by Bergemann and Morris (2008).

Definition 2.2. An environment $\mathcal{E}$ is economic in state $\theta$ if for any $a \in A$, there exist $i \neq j$ and alternatives $a_{i}$ and $a_{j}$ such that

$$
a_{i} \succ_{i}^{\theta} a \text { and } a_{j} \succ_{j}^{\theta} a \text {. }
$$

An environment $\mathcal{E}$ is economic if it is economic in every state $\theta \in \Theta$.

Observe that $\mathcal{E}$ is an economic environment if and only if in any state $\theta$, an alternative is most-preferred by at most $n-2$ players, i.e., $\bigcap_{i \in S} P_{1}(i, \theta)=\emptyset$ for all $S \subseteq N$ such that

\footnotetext{
${ }^{7}$ We take these state-dependent preference orderings over $\Delta A$ as primitives of the model instead of the state-dependent preference orderings over the set of probability measures on $A$ (set of probability measures on $A$ is in general a superset of $\Delta A$ ). This is because every outcome in the mechanisms that we study lies in $\Delta A$.
} 
$|S| \geq n-1$. This definition of economic environment is weaker than the usual definition in the literature. For instance, Serrano (2004) calls an environment as economic when, as part of the description of alternatives, there exists a private good like money over which players have strictly monotonic preferences (in such an environment, no two players have a common most-preferred alternative).

Benoit and Ok (2008) define the class of environments satisfying top-coincidence condition.

Definition 2.3. The environment $\mathcal{E}$ satisfies top-coincidence condition if for any $\theta \in \Theta$ and $S \subset N$ such that $|S|=n-1, \bigcap_{i \in S} P_{1}(i, \theta)$ is at most a singleton.

Thus, an environment that satisfies top-coincidence condition is such that in any state, any subset of $n-1$ players have at most one common most-preferred alternative. Clearly, any economic environment satisfies the top-coincidence condition. Therefore, the class of environments identified by top-coincidence condition is weaker than the class of economic environments.

We define two other classes of environments.

Definition 2.4. The environment $\mathcal{E}$ satisfies unique-top condition if for all $i \in N$ and $\theta \in \Theta$, the set of most-preferred alternatives $P_{1}(i, \theta)$ is singleton.

In an environment satisfying unique-top condition, every player has exactly one mostpreferred alternative in each state. An example of such an environment is when all players have strict preferences, i.e., for all $i \in N, \theta \in \Theta$, and $a, a^{\prime} \in A$, we have $a \sim_{i}^{\theta} a^{\prime} \Longleftrightarrow a=a^{\prime}$. Another example of an environment satisfying the unique-top condition is when every player's preference is single-peaked over $A$. It is easy to see that any environment that satisfies unique-top condition must also satisfy top-coincidence condition. However, there is no logical relation between the class of economic environments and the class of environments satisfying the unique-top condition, i.e., there exist both economic environments that do not satisfy the unique-top condition and noneconomic environments that satisfy the unique top condition.

Definition 2.5. Let $S \subseteq N$. The environment $\mathcal{E}$ satisfies diversity of top alternatives for $S(\mathrm{DTA}-S)$ if in any state, there does not exist an alternative that is unanimously mostpreferred by every player in $S$, i.e., $\bigcap_{i \in S} P_{1}(i, \theta)=\emptyset$ for any $\theta \in \Theta$.

For a given $S \subseteq N$, there are both economic and noneconomic environments that satisfy DTA- $S$. Moreover, if $|S| \geq n-1$, then every economic environment satisfies DTA-S. However, if $|S| \leq n-2$, then there exist economic environments that do not satisfy DTA-S. 


\subsection{Social Choice Rules}

Social goals are embodied in a social choice rule (SCR), which is a nonempty-valued correspondence $F: \Theta \rightarrow A$.

The following two properties of SCRs are prominent in the literature on Nash implementation.

Definition 2.6. SCR $F$ is Maskin monotonic if whenever an alternative $a \in F(\theta)$ and $a \notin F\left(\theta^{\prime}\right)$ for some $\theta$ and $\theta^{\prime}$, there exist player $i \in N$ and $a^{\prime} \in A$ such that

$$
a \succeq_{i}^{\theta} a^{\prime} \text { and } a^{\prime} \succ_{i}^{\theta^{\prime}} a
$$

Definition 2.7. SCR $F$ satisfies no-veto power if for any $\theta \in \Theta$ and $S \subseteq N$ such that $|S| \geq n-1$, we have

$$
\bigcap_{i \in S} P_{1}(i, \theta) \subseteq F(\theta)
$$

We will later introduce other notions of monotonicity and no-veto power among a subset of the players.

\subsection{Mechanism}

A social planner, who does not know the realized state, designs a mechanism in order to "implement" a SCR. The standard definition of a mechanism involves a set of messages for each player and an outcome function that maps a profile of messages of all players into the set of alternatives. We augment this definition with a political process that selects a senate, i.e., a subset of the players. Ultimately, the outcome is a function of only the messages of the senators. Formally, let $\mathcal{N}=2^{N} \backslash \emptyset$ be the set of all possible senates. Then a mechanism is a triplet $\Gamma=\left(\left(M_{i}\right)_{i \in N}, P,\left(g^{S}\right)_{S \in \mathcal{N}}\right)$, where

- $M_{i}$ is the set of opinions or messages that player $i$ can announce.

- $P$ is a political process for selecting a senate. If $S \subseteq N$ is the selected senate, then the messages of the players in $S,\left(m_{i}\right)_{i \in S}$, are transmitted to the planner and the messages of all players $j \notin S$ are ignored.

- $g^{S}: \prod_{i \in S} M_{i} \rightarrow A$ is the outcome function conditional on the selection of the senate $S \subseteq N$. Note that the outcome function $g^{S}$ is deterministic.

We let $m$ denote a typical element of $\prod_{i \in N} M_{i}$. For any $m \in \prod_{i \in N} M_{i}$ and $S \subseteq N$, let $m^{S}$ be the projection of $m$ into $\prod_{i \in S} M_{i}$ (note that if $S=N$, then $m^{N}=m$ ). That is, given 
any profile $m$ of messages of all players, $m^{S}$ is the profile of messages of only the players in $S$.

\subsection{Political Processes}

In this paper, we consider the following political processes: direct democracy, oligarchy, oligarchic democracy and random sampling. We define these processes next.

\subsubsection{Direct Democracy}

We define direct democracy, denoted by $D$, as the political process that selects $N$ as the senate with probability 1 . Hence, in direct democracy, every player's message is transmitted to the planner. A direct-democratic mechanism, $\Gamma_{D}=\left(\left(M_{i}\right)_{i \in N}, D,\left(g^{S}\right)_{S \in \mathcal{N}}\right)$, is a mechanism that uses direct democracy to select the senate. ${ }^{8}$

For any realization $\theta$, the direct-democratic mechanism $\Gamma_{D}$ defines the strategic-form game $\left\langle\Gamma_{D}, \theta\right\rangle$. In this game, each player $i$ announces a message $m_{i} \in M_{i}$. Since the political process is direct democracy, the messages of all the players are transmitted to the planner. The planner then implements the alternative $g^{N}(m)$. The players' preferences are given by the profile $\left(\succeq_{i}^{\theta}\right)_{i \in N}$. Let $N E\left(\Gamma_{D}, \theta\right)$ denote the set of Nash equilibria of $\left\langle\Gamma_{D}, \theta\right\rangle .{ }^{9}$

The literature has exclusively focused on direct-democratic mechanisms. The corresponding notion of implementation is called Nash implementation.

Definition 2.8. SCR $F$ is Nash implementable if there exists a direct-democratic mechanism $\Gamma_{D}=\left(\left(M_{i}\right)_{i \in N}, D,\left(g^{S}\right)_{S \in \mathcal{N}}\right)$ such that

$$
\left\{g^{N}(m): m \in N E\left(\Gamma_{D}, \theta\right)\right\}=F(\theta) \text { for all } \theta \in \Theta
$$

Maskin (1999) proves that Maskin monotonicity is necessary for Nash implementation.

Theorem 2.9 (Maskin (1999)). If SCR F is Nash implementable, then F is Maskin monotonic.

In general environments with at least three players, Maskin (1999) shows that Maskin monotonicity and no-veto power are sufficient for Nash implementation.

\footnotetext{
${ }^{8}$ Since messages of all players are transmitted to the planner, we only need to specify the outcome function $g^{N}$ as none of the other outcome functions are ever used in a direct democracy. However, we continue to list these functions in the definition of direct-democratic mechanisms just to make it consistent with the general definition of mechanisms given earlier. Similar remarks apply to other political processes.

${ }^{9}$ Like most of the literature on implementation, we restrict ourselves to pure strategies throughout this paper.
} 
Theorem 2.10 (Maskin (1999)). If $n \geq 3$, then any Maskin monotonic SCR that satisfies no-veto power is Nash implementable.

Since no-veto power is vacuously satisfied in economic environments, we have the following corollary:

Corollary 2.11. SCR $F$ is Nash implementable in an economic environment with $n \geq 3$ if and only if it is Maskin monotonic.

\subsubsection{Oligarchy}

Let $S$ be any proper subset of $N$. We define $S$ oligarchy, denoted by $O(S)$, as the political process that selects $S$ as the senate with probability 1. Hence, in $S$ oligarchy, the messages of all players in $S$ - the oligarchs - are transmitted to the planner whereas the messages of all players in $N \backslash S$ - the commoners - are ignored. An S-oligarchic mechanism, $\Gamma_{O(S)}=$ $\left(\left(M_{i}\right)_{i \in N}, O(S),\left(g^{S^{\prime}}\right)_{S^{\prime} \in \mathcal{N}}\right)$, is a mechanism that uses $S$ oligarchy to select the senate.

For any realization $\theta$, the $S$-oligarchic mechanism $\Gamma_{O(S)}$ defines the strategic-form game $\left\langle\Gamma_{O(S)}, \theta\right\rangle$. In this game, each player $i$ announces a message $m_{i} \in M_{i}$. Since the political process is $S$ oligarchy, the messages of all the oligarchs $S$ and only these players are transmitted to the planner. The planner then implements the alternative $g^{S}\left(m^{S}\right)$. The players' preferences are given by the profile $\left(\succeq_{i}^{\theta}\right)_{i \in N}$. Let $N E\left(\Gamma_{O(S)}, \theta\right)$ denote the set of Nash equilibria of $\left\langle\Gamma_{O(S)}, \theta\right\rangle$.

Definition 2.12. Let $S \subset N$. SCR $F$ is implementable by $S$ oligarchy if there exists a $S$-oligarchic mechanism $\Gamma_{O(S)}=\left(\left(M_{i}\right)_{i \in N}, O(S),\left(g^{S^{\prime}}\right)_{S^{\prime} \in \mathcal{N}}\right)$ such that

$$
\left\{g^{S}\left(m^{S}\right): m \in N E\left(\Gamma_{O(S)}, \theta\right)\right\}=F(\theta), \text { for all } \theta \in \Theta
$$

Remark 2.13. In a $S$-oligarchic mechanism, the commoners also announce their messages even though they know for sure that their messages will be ignored. A more reasonable assumption is that a player announces her message if and only if she knows that she is a senator. This alternative assumption can be easily incorporated into the model since for every $S$-oligarchic mechanism that implements $F$, there exists a corresponding game form that also implements $F$ but asks only the oligarchs to announce their messages. To see this, fix an $S$-oligarchic mechanism $\Gamma_{O(S)}$ that implements $F$. Consider the following game form: all the oligarchs $S$ simultaneously announce their messages $\left(m_{i}\right)_{i \in S} \in \prod_{i \in S} M_{i}$, which are transmitted to the planner who then implements $g^{S}\left(\left(m_{i}\right)_{i \in S}\right)$. Clearly, this game form also implements $F$ in Nash equilibrium. 


\subsubsection{Oligarchic Democracy}

By $S$-oligarchic democracy, we mean the political process in which the senate is either a fixed $S \subset N$ or $N$ but the probability it is $N$ is arbitrarily close to 0 . To capture the idea of "arbitrarily close to 0 ", we define a sequence of political processes in which the probability of selecting $N$ as the senate is converging to 0 .

Formally, for any $S \subset N$ and $\epsilon \in(0,1)$, let $(S, \epsilon)$-oligarchic democracy, denoted by $O(S, \epsilon)$, be the political process that selects $S$ as the senate with probability $1-\epsilon$ and $N$ as the senate with probability $\epsilon$. Hence, in $(S, \epsilon)$-oligarchic democracy, either the messages of only the players in $S$ - the oligarchs - are transmitted to the planner with probability $1-\epsilon$ or the messages of all players in $N$ are transmitted to the planner with probability $\epsilon$. An $(S, \epsilon)$-oligarchic-democratic mechanism, $\Gamma_{O(S, \epsilon)}=\left(\left(M_{i}\right)_{i \in N}, O(S, \epsilon),\left(g^{S^{\prime}}\right)_{S^{\prime} \in \mathcal{N}}\right)$, is a mechanism that uses $(S, \epsilon)$-oligarchic democracy to select the senate.

For any realization $\theta$, the $(S, \epsilon)$-oligarchic-democratic mechanism $\Gamma_{O(S, \epsilon)}$ defines a strategicform game $\left\langle\Gamma_{O(S, \epsilon)}, \theta\right\rangle$. In this game, each player $i$ announces a message $m_{i} \in M_{i}$. Since the political process is $(S, \epsilon)$-oligarchic democracy, either the messages of all the oligarchs $S$ are transmitted to the planner with probability $1-\epsilon$ or the messages of all the players in $N$ are transmitted to the planner with probability $\epsilon$. If the planner only receives the messages of the oligarchs $S$, then she implements the alternative $g^{S}\left(m^{S}\right)$; on the other hand, if the planner receives the messages of all the players in $N$, then she implements the alternative $g^{N}(m)$. This induces the lottery $l[S, \epsilon, m]$ in $\Delta A$ such that $l[S, \epsilon, m]$ assigns probability $1-\epsilon$ to alternative $g^{S}\left(m^{S}\right)$ and probability $\epsilon$ to alternative $g^{N}(m)$. The players' preferences are given by the profile $\left(\succeq_{i}^{\theta}\right)_{i \in N}$. Let $N E\left(\Gamma_{O(S, \epsilon)}, \theta\right)$ denote the set of Nash equilibria of $\left\langle\Gamma_{O(S, \epsilon)}, \theta\right\rangle$.

Definition 2.14. Let $S \subset N$. SCR $F$ is implementable by $S$-oligarchic democracy if there exists a sequence of $\left(S, \epsilon_{k}\right)$-oligarchic-democratic mechanisms

$$
\left(\Gamma_{O\left(S, \epsilon_{k}\right)}=\left(\left(M_{i}\right)_{i \in N}, O\left(S, \epsilon_{k}\right),\left(g^{S^{\prime}}\right)_{S^{\prime} \in \mathcal{N}}\right)\right)_{k=1}^{\infty}
$$

such that for all $k$,

(i) $\epsilon_{k} \in(0,1)$ with $\lim _{k \rightarrow \infty} \epsilon_{k} \rightarrow 0$ and

(ii) $\left\{l\left[S, \epsilon_{k}, m\right]: m \in N E\left(\Gamma_{O\left(S, \epsilon_{k}\right)}, \theta\right)\right\}=F(\theta)$, for all $\theta \in \Theta$.

Condition (i) in the above definition formalizes the idea that the probability of selecting $N$ as the senate is "arbitrarily close to 0 ". Condition (ii) has two requirements. First, if $m \in$ $N E\left(\Gamma_{O\left(S, \epsilon_{k}\right)}, \theta\right)$, then we must have $l\left[S, \epsilon_{k}, m\right] \in F(\theta)$, i.e., $l\left[S, \epsilon_{k}, m\right]$ is deterministic with $g^{S}\left(m^{S}\right)=g^{N}(m) \in F(\theta)$. Second, for any $a \in F(\theta)$, there must exist $m \in N E\left(\Gamma_{O\left(S, \epsilon_{k}\right)}, \theta\right)$ 
such that $l\left[S, \epsilon_{k}, m\right]=a$. Hence, although lotteries are generated in the selection of the senators, implementation by $S$-oligarchic democracy requires exact implementation of the SCR by every $\left(S, \epsilon_{k}\right)$-oligarchic-democratic mechanism.

Remark 2.15. The alternative assumption that a player announces her message if and only if she is a senator can also be incorporated into oligarchic democracy. Fix a $(S, \epsilon)$ oligarchic-democratic mechanism that implements $F$. Consider the following extensive-form game: first, the oligarchs $S$ announce their messages $\left(m_{i}\right)_{i \in S} \in \prod_{i \in S} M_{i}$. Then, with probability $1-\epsilon$, the game ends and planner implements $g^{S}\left(\left(m_{i}\right)_{i \in S}\right)$; but with probability $\epsilon$, the planner asks the commoners to announce their messages $\left(m_{j}\right)_{j \in N \backslash S} \in \prod_{j \in N \backslash S} M_{j}$ and then implements $g^{N}\left(\left(m_{i}\right)_{i \in S},\left(m_{j}\right)_{j \in N \backslash S}\right)$. A crucial assumption is that the commoners do not observe the oligarchs' messages when they are asked to announce their messages. In this extensive-form game, every player moves at exactly one information set and thus, it has the same strategic form as $\Gamma_{O(S, \epsilon)}$. Hence, the extensive-form game also implements $F$ in Nash equilibrium. ${ }^{10}$

\subsubsection{Random Sampling}

The final political process that we consider is random sampling. In this process, denoted by $R(\bar{n})$, the planner randomly chooses a sample of the messages of the players, where the sample size is fixed at some positive integer $\bar{n}<n$. Let $\mathcal{N}_{\bar{n}}$ denote the set of all senates $S \in \mathcal{N}$ such that $|S|=\bar{n}$. Thus, in random sampling, the senate is equally likely to be any $S \in \mathcal{N}_{\bar{n}}$. The $R(\bar{n})$-sampling mechanism, $\Gamma_{R(\bar{n})}=\left(\left(M_{i}\right)_{i \in N}, R(\bar{n}),\left(g^{S}\right)_{S \in \mathcal{N}}\right)$, is a mechanism that uses $R(\bar{n})$ sampling procedure to select the senate.

For any realization $\theta$, the $R(\bar{n})$-sampling mechanism $\Gamma_{R(\bar{n})}$ defines a strategic-form game $\left\langle\Gamma_{R(\bar{n})}, \theta\right\rangle$. In this game, each player $i$ announces a message $m_{i} \in M_{i}$. Since the political process is $R(\bar{n})$, a subset $S \in \mathcal{N}_{\bar{n}}$ is chosen with probability $1 /\left|\mathcal{N}_{\bar{n}}\right|$ and the messages of all the players in $S$ are transmitted to the planner. The planner then implements the alternative $g^{S}\left(m^{S}\right)$. Thus, the probability that the alternative $g^{S}\left(m^{S}\right)$ is implemented is equal to $1 /\left|\mathcal{N}_{\bar{n}}\right|$. Let $l[R(\bar{n}), m]$ be the lottery in $\Delta A$, which for all $S \in \mathcal{N}_{\bar{n}}$, assigns probability $1 /\left|\mathcal{N}_{\bar{n}}\right|$ to alternative $g^{S}\left(m^{S}\right)$. The players' preferences are given by the profile $\left(\succeq_{i}^{\theta}\right)_{i \in N}$. Let $N E\left(\Gamma_{R(\bar{n})}, \theta\right)$ denote the set of Nash equilibria of $\left\langle\Gamma_{R(\bar{n})}, \theta\right\rangle$.

Definition 2.16. SCR $F$ is implementable by $\bar{n}$-random sampling if there exists a $R(\bar{n})$ -

\footnotetext{
${ }^{10}$ In fact, we can argue that in each state, the set of Perfect Bayesian equilibria of the extensive-form game coincides with the set of Nash equilibria of $\Gamma_{O(S, \epsilon)}$. This is because all players have the same incentives at their respective information sets in both game forms. This is clearly true for the oligarchs. Although the commoners find out that they are in the senate if they are asked to play the game yet, since preferences over lotteries are monotone, their incentives in the extensive-form game are the same as in $\Gamma_{O(S, \epsilon)}$.
} 
sampling mechanism $\Gamma_{R(\bar{n})}=\left(\left(M_{i}\right)_{i \in N}, R(\bar{n}),\left(g^{S}\right)_{S \in \mathcal{N}}\right)$ such that

$$
\left\{l[R(\bar{n}), m]: m \in N E\left(\Gamma_{R(\bar{n})}, \theta\right)\right\}=F(\theta) \text {, for all } \theta \in \Theta
$$

Thus, just like in the model of oligarchic democracy, implementation by $\bar{n}$-random sampling requires exact implementation of the SCR even though a lottery is used in the sampling of the messages.

Remark 2.17. The alternative assumption that a player announces her message if and only if she is a senator can also be incorporated into random sampling. Fix a $R(\bar{n})$-sampling mechanism that implements $F$. Consider the following extensive-form game: first, a senate of size $\bar{n}$ is randomly chosen but the selected senators $S$ are not informed of each other's identities. Then the senators $S$ announce their messages $\left(m_{i}\right)_{i \in S} \in \prod_{i \in S} M_{i}$ and $g^{S}\left(\left(m_{i}\right)_{i \in S}\right)$ is implemented. In this extensive-form game, every player moves at exactly one information set and thus, it has the same strategic form as $\Gamma_{R(\bar{n})}$. Hence, the extensive-form game also implements $F$ in Nash equilibrium. ${ }^{11}$

\section{$3 \quad$ Implementation by Oligarchy}

Consider any set of oligarchs $S \subset N$. It is easy to see that a $S$ oligarchy in environment $\mathcal{E}=\left\langle N, A,\left(\left(\succeq_{i}^{\theta}\right)_{i \in N}\right)_{\theta \in \Theta}\right\rangle$ is equivalent to a direct democracy in the restricted environment $\mathcal{E}(S)=\left\langle S, A,\left(\left(\succeq_{i}^{\theta}\right)_{i \in S}\right)_{\theta \in \Theta}\right\rangle$. Hence, the necessary and sufficient conditions for implementation by $S$ oligarchy in environment $\mathcal{E}$ will be equivalent to the corresponding conditions for Nash implementation in environment $\mathcal{E}(S)$.

Restricting attention to a set of oligarchs $S$, we can define notions of monotonicity and no-veto power with respect to $S$ as follows:

Definition 3.1. Let $S \subset N$. SCR $F$ is $S$-monotonic if whenever $a \in F(\theta)$ and $a \notin F\left(\theta^{\prime}\right)$ for some $a, \theta$ and $\theta^{\prime}$, there exist player $i \in S$ and $a^{\prime} \in A$ such that

$$
a \succeq_{i}^{\theta} a^{\prime} \text { and } a^{\prime} \succ_{i}^{\theta^{\prime}} a
$$

\footnotetext{
${ }^{11}$ Under the stronger assumption that players' preferences over lotteries satisfy the independence axiom, we can argue that in each state, the set of Perfect Bayesian equilibria of the extensive-form game coincides with the set of Nash equilibria of $\Gamma_{R(\bar{n})}$. When a player is asked to play, she finds out that she is in the senate but she does not know who else is in the senate. However, due to the independence axiom, the player's incentives in the extensive-form game are the same as in $\Gamma_{R(\bar{n})}$.
} 
Definition 3.2. Let $S \subset N$. SCR $F$ satisfies $S$-no-veto power if for any $\theta \in \Theta$ and $S^{\prime} \subseteq S$ such that $\left|S^{\prime}\right| \geq|S|-1$, we have

$$
\bigcap_{i \in S^{\prime}} P_{1}(i, \theta) \subseteq F(\theta)
$$

We easily obtain the following corollaries from, respectively, Theorems 2.9 and 2.10:

Corollary 3.3. If SCR $F$ is implementable by $S$ oligarchy, then $F$ is $S$-monotonic.

Corollary 3.4. If $3 \leq|S|<n$, then any $S C R$ that satisfies $S$-monotonicity and $S$-no-veto power is implementable by $S$ oligarchy.

$S$-no-veto power is vacuously true if $\mathcal{E}(S)$ is an economic environment. Hence, we have the following result:

Corollary 3.5. Suppose $3 \leq|S|<n$ and $\mathcal{E}(S)$ is an economic environment. $S C R F$ is implementable by $S$ oligarchy if and only if it is $S$-monotonic.

\subsection{Comparison}

Clearly, $S$-monotonicity is stronger than Maskin monotonicity. Furthermore, we have the following result:

Proposition 3.6. If an $S C R F$ is implementable by $S$ oligarchy for some $S \subset N$, then $F$ is Nash implementable.

Proof. Let $\Gamma_{O(S)}=\left(\left(M_{i}\right)_{i \in N}, O(S),\left(g^{S^{\prime}}\right)_{S^{\prime} \in \mathcal{N}}\right)$ be the $S$-oligarchic mechanism that implements $F$. Define the direct democracy $\Gamma_{D}=\left(\left(M_{i}\right)_{i \in N}, D,\left(\hat{g}^{S^{\prime}}\right)_{S^{\prime} \in \mathcal{N}}\right)$ such that $\hat{g}^{N}(m)=$ $g^{S}\left(m^{S}\right), \forall m \in M$, and $\hat{g}^{S^{\prime}}=g^{S^{\prime}}, \forall S^{\prime} \neq N$. It is easy to show that for all $\theta$, we have $m \in N E\left(\Gamma_{O(S)}, \theta\right) \Longleftrightarrow m \in N E\left(\Gamma_{D}, \theta\right)$, and hence, the result follows.

Thus, in any environment, any SCR that is implementable by an oligarchy is also implementable by direct democracy (i.e., Nash implementable). However, the converse is not true. That is, there are SCRs that are Nash implementable but not implementable by $S$ oligarchy for any $S \subset N$. Consider the following example:

Example 3.7. Let $N=\{1,2,3,4\}, A=\{a, b, c, d\}$ and $\Theta=\left\{\theta, \theta^{\prime}\right\}$. The preferences of the 
four players over alternatives in the two states are as follows:

$$
\begin{array}{cc}
\theta & \theta^{\prime} \\
a \succ_{1}^{\theta} b \succ_{1}^{\theta} c \succ_{1}^{\theta} d & b \succ_{1}^{\theta^{\prime}} a \succ_{1}^{\theta^{\prime}} c \succ_{1}^{\theta^{\prime}} d \\
b \succ_{2}^{\theta} c \succ_{2}^{\theta} d \succ_{2}^{\theta} a & c \succ_{2}^{\theta^{\prime}} b \succ_{2}^{\theta^{\prime}} d \succ_{2}^{\theta^{\prime}} a \\
c \sim_{3}^{\theta} d \succ_{3}^{\theta} a \succ_{3}^{\theta} b & d \succ_{3}^{\theta^{\prime}} c \succ_{3}^{\theta^{\prime}} a \succ_{3}^{\theta^{\prime}} b \\
a \succ_{4}^{\theta} d \succ_{4}^{\theta} b \succ_{4}^{\theta} c & d \sim_{4}^{\theta^{\prime}} a \succ_{4}^{\theta^{\prime}} b \succ_{4}^{\theta^{\prime}} c
\end{array}
$$

Consider the SCR $F$ such that $F(\theta)=\{a, b, c\}$ and $F\left(\theta^{\prime}\right)=\{d\}$. Player 1 is the only player $i$ for whom there exists an alternative $\hat{a}$ such that $a \succeq_{i}^{\theta} \hat{a}$ and $\hat{a} \succ_{i}^{\theta^{\prime}} a$. Player 2 is the only player $i$ for whom there exists an alternative $\hat{a}$ such that $b \succeq_{i}^{\theta} \hat{a}$ and $\hat{a} \succ_{i}^{\theta^{\prime}} b$. Player 3 is the only player $i$ for whom there exists an alternative $\hat{a}$ such that $c \succeq_{i}^{\theta} \hat{a}$ and $\hat{a} \succ_{i}^{\theta^{\prime}} c$. Finally, player 4 is the only player $i$ for whom there exists an alternative $\hat{a}$ such that $d \succeq_{i}^{\theta^{\prime}} \hat{a}$ and $\hat{a} \succ_{i}^{\theta} d$. Therefore, $F$ is Maskin monotonic but not $S$-monotonic for any $S \subset N$. Since the environment is economic, it follows that $F$ is Nash implementable but it is not implementable by any $S$ oligarchy.

\section{Implementation by Oligarchic Democracy}

\subsection{Necessary Condition}

Definition 4.1. Let $S \subset N$. SCR $F$ is weak $S$-monotonic if there exists a sequence $\left(\epsilon_{k}\right)_{k=1}^{\infty}$ with $\epsilon_{k} \in(0,1), \forall k$, and $\lim _{k \rightarrow \infty} \epsilon_{k}=0$ such that whenever $a \in F(\theta)$ and $a \notin F\left(\theta^{\prime}\right)$ for some $a, \theta$ and $\theta^{\prime}$ then for all $k$ either:

(i) there exist player $i_{k} \in N \backslash S$ and alternative $a_{k}^{\prime}$ such that $a \succeq_{i_{k}}^{\theta} a_{k}^{\prime}$ and $a_{k}^{\prime} \succ_{i_{k}}^{\theta^{\prime}} a$ or

(ii) there exist player $i_{k} \in S$, lottery $l_{k}$, and alternatives $\hat{a}_{k}$ and $\tilde{a}_{k}$ such that

$$
l_{k}\left(\hat{a}_{k}\right)=\epsilon_{k}, \quad l_{k}\left(\tilde{a}_{k}\right)=1-\epsilon_{k}, \quad a \succeq_{i_{k}}^{\theta} l_{k} \text { and } l_{k} \succ_{i_{k}}^{\theta^{\prime}} a .
$$

Thus, weak $S$-monotonicity requires that there exists a sequence of positive probabilities $\epsilon_{k}$ converging to 0 such that whenever an alternative $a$ is in the SCR for some state $\theta$ but drops out of the SCR in state $\theta^{\prime}$, then in going from $\theta$ to $\theta^{\prime}$, there must exist either (i) some commoner, for whom there is reversal of her preferences over alternatives around $a$ (i.e., there is another alternative that she weakly prefers to $a$ in $\theta$ but this is not true in $\theta^{\prime}$ ) or (ii) some oligarch, for whom there is reversal of her preferences over lotteries around $a$ (i.e., there is a lottery that she weakly prefers to $a$ in $\theta$ but this is not true in $\theta^{\prime}$ ), where we 
consider only those lotteries that have at most two alternatives in their supports and assign probability of $\epsilon_{k}$ to one of those alternatives.

As we show in the next result, weak $S$-monotonicity is a necessary condition for implementation by $S$-oligarchic democracy.

Theorem 4.2. If $S C R F$ is implementable by $S$-oligarchic democracy, then $F$ is weak $S$ monotonic.

Proof. Let $\left(\Gamma_{O\left(S, \epsilon_{k}\right)}=\left(\left(M_{i}\right)_{i \in N}, O\left(S, \epsilon_{k}\right),\left(g^{S^{\prime}}\right)_{S^{\prime} \in \mathcal{N}}\right)\right)_{k=1}^{\infty}$ be the sequence of $\left(S, \epsilon_{k}\right)$-oligarchicdemocratic mechanisms with $\epsilon_{k} \in(0,1), \forall k$, and $\lim _{k \rightarrow \infty} \epsilon_{k} \rightarrow 0$, which implement $F$ by $S$-oligarchic democracy. We show that $F$ is weak $S$-monotonic.

Consider the sequence $\left(\epsilon_{k}\right)_{k=1}^{\infty}$. Suppose there exist $a, \theta$ and $\theta^{\prime}$ such that $a \in F(\theta)$ but $a \notin F\left(\theta^{\prime}\right)$. Then for all $k$, there must exist $m^{k} \in N E\left(\Gamma_{O\left(S, \epsilon_{k}\right)}, \theta\right)$ with $l\left[S, \epsilon_{k}, m^{k}\right]=a$, i.e., $g^{N}\left(m^{k}\right)=g^{S}\left(m^{k S}\right)=a$. Since $a \notin F\left(\theta^{\prime}\right)$, it must be that $m^{k} \notin N E\left(\Gamma_{O\left(S, \epsilon_{k}\right)}, \theta^{\prime}\right)$. Hence, there must exist $i_{k} \in N$ and $m_{i_{k}} \in M_{i_{k}}$ such that

$$
a \succeq_{i_{k}}^{\theta} l\left[S, \epsilon_{k},\left(m_{i_{k}}, m_{-i_{k}}^{k}\right)\right] \text { and } l\left[S, \epsilon_{k},\left(m_{i_{k}}, m_{-i_{k}}^{k}\right)\right] \succ_{i_{k}}^{\theta^{\prime}} a
$$

If $i_{k} \in N \backslash S$, then $g^{S}\left(\left(m_{i_{k}}, m_{-i_{k}}^{k}\right)^{S}\right)=a$. Hence, in this case, $l\left[S, \epsilon_{k},\left(m_{i_{k}}, m_{-i_{k}}^{k}\right)\right]$ is a lottery which assigns probability $\epsilon_{k}$ to alternative $g^{N}\left(m_{i_{k}}, m_{-i_{k}}^{k}\right)=a_{k}^{\prime}$ and probability $1-\epsilon_{k}$ to alternative $a$. Since the preferences over lotteries are monotone, we have $a \succeq_{i_{k}}^{\theta} a_{k}^{\prime}$ and $a_{k}^{\prime} \succ_{i_{k}}^{\theta^{\prime}} a$. Thus, the first condition in the definition of weak $S$-monotonicity is satisfied.

If $i_{k} \in S$, then $l\left[S, \epsilon_{k},\left(m_{i_{k}}, m_{-i_{k}}^{k}\right)\right]$ is a lottery which assigns probability $\epsilon_{k}$ to alternative $g^{N}\left(m_{i_{k}}, m_{-i_{k}}^{k}\right)=\hat{a}_{k}$ and probability $1-\epsilon_{k}$ to $g^{S}\left(\left(m_{i_{k}}, m_{-i_{k}}^{k}\right)^{S}\right)=\tilde{a}_{k}$ with $a \succeq_{i_{k}}^{\theta}$ $l\left[S, \epsilon_{k},\left(m_{i_{k}}, m_{-i_{k}}^{k}\right)\right]$ and $l\left[S, \epsilon_{k},\left(m_{i_{k}}, m_{-i_{k}}^{k}\right)\right] \succ_{i_{k}}^{\theta^{\prime}} a$. Hence, in this case, the second condition in the definition of weak $S$-monotonicity is satisfied.

The above proof also makes it clear why we require a reversal of preferences over alternatives for a commoner while a reversal of preferences over lotteries with at most two alternatives in their supports for oligarchs. In $S$-oligarchic democracy, the messages of the commoners are only considered in case $N$ is selected as the senate. As a result, by changing her message in an oligarchic-democratic mechanism, a commoner can at most change the alternative that is implemented when the senate is $N$. Hence, whenever a commoner has an improving unilateral deviation, it must be that she is able to generate a strictly preferred alternative when the senate is $N$. On the other hand, the messages of the oligarchs are considered irrespective of whether $S$ or $N$ is the senate. Hence, by changing her message in an oligarchic-democratic mechanism, an oligarch can, in principle, change any of the alternatives that are implemented when the senate is $S$ and when the senate is $N$. That is why, 
whenever an oligarch has an improving unilateral deviation, it must be that she is able to generate a strictly preferred lottery with at most two alternatives in its support.

Note that the reversal of preferences over lotteries around an alternative is a weaker requirement than the reversal of preferences over alternatives around an alternative. Thus, we have the following lemma (proof is omitted):

Lemma 4.3. If $F$ is Maskin monotonic, then $F$ is weak $S$-monotonic for all $S \subset N$.

\subsection{Economic Environments}

\subsubsection{Sufficient Condition}

It turns out, if there are at least three oligarchs in $S$, then weak $S$-monotonicity of an SCR is also a sufficient condition for its implementation by $S$-oligarchic democracy in economic environments.

Theorem 4.4. Suppose $3 \leq|S|<n$ and $\mathcal{E}$ is an economic environment. If $S C R F$ is weak $S$-monotonic, then $F$ is implementable by $S$-oligarchic democracy.

Sketch of the proof: Consider the sequence $\left(\epsilon_{k}\right)_{k=1}^{\infty}$ with respect to which $F$ satisfies the definition of weak $S$-monotonicity. For all $k$, define the $\left(S, \epsilon_{k}\right)$-oligarchic-democratic mechanism $\Gamma_{O\left(S, \epsilon_{k}\right)}=\left(\left(M_{i}\right)_{i \in N}, O\left(S, \epsilon_{k}\right),\left(g^{S^{\prime}}\right)_{S^{\prime} \in \mathcal{N}}\right)$ such that for all $i \in N$,

$$
M_{i}=\Theta \times A \times A \times A \times \mathbb{Z}_{+}
$$

where $\mathbb{Z}_{+}$is the set of nonnegative integers. Let a typical message $m_{i}$ be of the form $\left(\theta_{i}, a_{i}^{1}, a_{i}^{2}, a_{i}^{3}, z_{i}\right)$. The outcome function $g^{N}$ is as follows:

(i) If for every player $i \in N, m_{i}=\left(\theta, a_{i}^{1}, a, a, 0\right)$ and $a \in F(\theta)$, then $g^{N}(m)=a$.

(ii) If for $n-1$ players $i \neq j$ in $N, m_{i}=\left(\theta, a_{i}^{1}, a, a, 0\right)$ and $a \in F(\theta)$, but $m_{j}=$ $\left(\theta_{j}, a_{j}^{1}, a_{j}^{2}, a_{j}^{3}, z_{j}\right) \neq\left(\theta, a_{j}^{1}, a, a, 0\right)$, then

$$
\begin{aligned}
& j \in N \backslash S \Longrightarrow g^{N}(m)=\left\{\begin{array}{cl}
a & \text { if } a_{j}^{2} \succ_{j}^{\theta} a \\
a_{j}^{2} & \text { if } a \succeq_{j}^{\theta} a_{j}^{2},
\end{array}\right. \\
& j \in S \Longrightarrow g^{N}(m)=\left\{\begin{array}{cl}
a & \text { if } l_{k}[j] \succ_{j}^{\theta} a \\
a_{j}^{2} & \text { if } a \succeq_{j}^{\theta} l_{k}[j],
\end{array}\right.
\end{aligned}
$$

where $l_{k}[j]$ is the lottery that assigns probability $\epsilon_{k}$ to $a_{j}^{2}$ and probability $1-\epsilon_{k}$ to $a_{j}^{3}$. 
(iii) In all other cases, $g^{N}(m)=a_{j}^{1}$ where $j \in N$ is the player with the lowest index among those who announce the highest integer. ${ }^{12}$

The outcome function $g^{S}$ is as follows:

(i') If for every player $i \in S, m_{i}=\left(\theta, a_{i}^{1}, a, a, 0\right)$ and $a \in F(\theta)$, then $g^{S}\left(\left(m_{i}\right)_{i \in S}\right)=a$.

(ii') If for $|S|-1$ players $i \neq j$ in $S, m_{i}=\left(\theta, a_{i}^{1}, a, a, 0\right)$ and $a \in F(\theta)$, but $m_{j}=$ $\left(\theta_{j}, a_{j}^{1}, a_{j}^{2}, a_{j}^{3}, z_{j}\right) \neq\left(\theta, a_{j}^{1}, a, a, 0\right)$, then

$$
g^{S}\left(\left(m_{i}\right)_{i \in S}\right)=\left\{\begin{array}{cl}
a & \text { if } l_{k}[j] \succ_{j}^{\theta} a . \\
a_{j}^{3} & \text { if } a \succeq_{j}^{\theta} l_{k}[j] .
\end{array}\right.
$$

(iii') In all other cases, $g^{S}\left(\left(m_{i}\right)_{i \in S}\right)=a_{j}^{1}$ where $j \in S$ is the player with the lowest index among those players in $S$ who announce the highest integer in the profile $\left(m_{i}\right)_{i \in S}$.

Finally, for all $S^{\prime} \in \mathcal{N} \backslash\{N, S\}$, the outcome function $g^{S^{\prime}}$ can be arbitrary.

Thus, each player sends a message with five components $\left(\theta_{i}, a_{i}^{1}, a_{i}^{2}, a_{i}^{3}, z_{i}\right)$. The outcome functions $g^{N}$ and $g^{S}$ are defined like in the canonical mechanism of Maskin (1999) with three rules. The first rules are used when there is agreement in all the messages received by the planner, except possibly in the second components $a_{i}^{1}$. In this case, the planner implements the commonly agreed alternative. The second rules allow the planner to use weak $S$-monotonicity of the SCR to eliminate the possibility that players agree on a message with a "bad" alternative. In such a situation, rule (ii) gives any commoner $j$ the opportunity to deviate and implement a strictly preferred alternative $a_{j}^{2}$ when the senate is $N$ by using the reversal of her preferences over alternatives around the "bad" alternative. Although the probability that $a_{j}^{2}$ will be implemented is arbitrarily small, the fact that it is positive and deviations by commoner $j$ do not affect the alternative that is implemented when the senate is $S$ provide sufficient incentives for $j$ to deviate. Similarly, whenever the players agree on a "bad" alternative, rules (ii) and (ii') give any oligarch $j$ the opportunity to deviate and

\footnotetext{
${ }^{12}$ The advantage of the integer game in our framework is that by announcing a high enough integer, a player can win the integer game in every senate. Notice that this cannot be achieved by using the modulo game in which each player announces a single integer. A particular integer might make the player win the modulo game in one senate but she could at the same time lose the modulo game in some other senate. Hence, in order to replace the integer game with the modulo game, we need each player to announce as many integers between 1 and $n$ as the number of senates she can be a member of. Thus, in oligarchic democracy, oligarchs must announce two integers. The mechanisms used in the proofs for oligarchic democracy can be altered in this fashion without changing the results. In contrast, the proof of the main result in the random-sampling model relies heavily on the existence of a unique player who has the lowest index among those who announce the highest integer in the whole population. Thus, replacing the integer game with the modulo game seems difficult in that model. However, the use of both these games in implementation has been criticized; see Jackson (1992).
} 
implement a strictly preferred lottery by using the reversal of her preferences over lotteries, which have at most two alternatives in their supports, around the "bad" alternative. This is done by implementing $a_{j}^{2}$ in case the senate is $N$, and $a_{j}^{3}$ when the senate is $S$. Finally, the third rules eliminate the possibility of any equilibria other than those in which all players agree in their messages. In these situations, at least $n-1$ players are such that each of them can implement her most-preferred alternative when the senate is $N$ by announcing such an alternative in the second component of her message and a high enough integer. However, such a deviation will be improving if it does not cause a worse alternative to be implemented when the senate is $S$. This is obviously true for the commoners. On the other hand, since rule (ii') does not depend on the second and the last components of the deviating oligarch's message, every oligarch also ensures that a worse alternative is not implemented when the senate is $S$. As a result, we obtain a contradiction with the assumption of economic environment. These arguments are formalized in the proof presented in the Appendix. $\diamond$

\subsubsection{Comparison}

We obtain the following result as a corollary of Theorem 2.9, Lemma 4.3 and Theorem 4.4:

Corollary 4.5. Suppose $3 \leq|S|<n$ and $\mathcal{E}$ is an economic environment. If $F$ is Nash implementable, then $F$ is implementable by S-oligarchic democracy.

Thus, in economic environments, any SCR that is implementable by direct democracy (i.e., Nash implementable) is also implementable by any $S$-oligarchic democracy that has at least three oligarchs. In particular, any three players can be designated as the oligarchs in order to implement any SCR in economic environments using oligarchic democracy.

The next example shows that there are SCRs that are implementable by oligarchic democracies but not Nash implementable. Hence, weak $S$-monotonicity is strictly weaker than Maskin monotonicity.

Example 4.6 (Strong Pareto Correspondence). Let $N=\{1,2,3,4\}, A=\{a, b, c, d\}$ and $\Theta=\left\{\theta, \theta^{\prime}\right\}$. The preferences of the four players over alternatives in the two states are as follows:

$$
\begin{array}{cc}
\theta & \theta^{\prime} \\
a \succ_{1}^{\theta} b \succ_{1}^{\theta} c \succ_{1}^{\theta} d & a \succ_{1}^{\theta^{\prime}} b \sim_{1}^{\theta^{\prime}} c \succ_{1}^{\theta^{\prime}} d \\
b \succ_{2}^{\theta} c \succ_{2}^{\theta} d \succ_{2}^{\theta} a & b \sim_{2}^{\theta^{\prime}} c \sim_{2}^{\theta^{\prime}} d \sim_{2}^{\theta^{\prime}} a \\
c \succ_{3}^{\theta} d \succ_{3}^{\theta} a \succ_{3}^{\theta} b & d \succ_{3}^{\theta^{\prime}} c \succ_{3}^{\theta^{\prime}} a \succ_{3}^{\theta^{\prime}} b \\
a \succ_{4}^{\theta} c \sim_{4}^{\theta} d \succ_{4}^{\theta} b & c \succ_{4}^{\theta^{\prime}} a \sim_{4}^{\theta^{\prime}} d \succ_{4}^{\theta^{\prime}} b
\end{array}
$$

Furthermore, we assume that players' preferences over lotteries are represented by expected utility. 
Let SCR $F$ be the strong Pareto correspondence, i.e., for all $\theta^{\prime \prime} \in \Theta$,

$$
F\left(\theta^{\prime \prime}\right)=\left\{\hat{a} \in A: \nexists a^{\prime} \in A \text { s.t. } \forall i \in N, a^{\prime} \succeq_{i}^{\theta^{\prime \prime}} \hat{a} \text { and } \exists j \in N \text { with } a^{\prime} \succ_{j}^{\theta^{\prime \prime}} \hat{a}\right\}
$$

It is easy to see that $F(\theta)=\{a, b, c\}$ and $F\left(\theta^{\prime}\right)=\{a, c, d\} . F$ is not Maskin monotonic since $b \in F(\theta), b \notin F\left(\theta^{\prime}\right)$ but there does not exist any player $i$ and alternative $\hat{a}$ such that $b \succeq_{i}^{\theta} \hat{a}$ and $\hat{a} \succ_{i}^{\theta^{\prime}} b$. Therefore, $F$ is not Nash implementable.

We argue that $F$ is weak $S$-monotonic for any $S \subset N$ such that $1 \in S$. Since player 1 has expected-utility preferences, $a \succ_{1}^{\theta} b \succ_{1}^{\theta} c$ and $a \succ_{1}^{\theta^{\prime}} b \sim_{1}^{\theta^{\prime}} c$ imply that there exists a small enough $\epsilon^{\prime} \in(0,1)$ such that for all $\epsilon \in\left(0, \epsilon^{\prime}\right]$, we have

$$
b \succeq_{1}^{\theta} l_{\epsilon} \text { and } l_{\epsilon} \succ_{1}^{\theta^{\prime}} b,
$$

where $l_{\epsilon}$ is the lottery with $l_{\epsilon}(a)=\epsilon$ and $l_{\epsilon}(c)=1-\epsilon$. Moreover, for alternative $d$, which is such that $d \in F\left(\theta^{\prime}\right)$ but $d \notin F(\theta)$, we have player 3 with $d \succ_{3}^{\theta^{\prime}} c$ and $c \succ_{3}^{\theta} d$. Therefore, if $1 \in S$, then for any sequence $\left(\epsilon_{k}\right)_{k=1}^{\infty}$ such that $\epsilon_{k} \in\left(0, \epsilon^{\prime}\right]$ and $\lim _{k \rightarrow \infty} \epsilon_{k}=0, F$ satisfies the conditions for weak $S$-monotonicity.

It is easy to see that the environment is economic. Hence, Theorem 4.4 implies that $F$ is implementable by $S$-oligarchic democracy, where $1 \in S$ and $|S|=3$.

Here, we note that the strong Pareto correspondence is not necessarily weak $S$-monotonic in every economic environment. Still, as the above example illustrates, compared to Nash implementation, we can implement the strong Pareto correspondence by $S$-oligarchic democracy in a larger set of economic environments.

\subsection{Noneconomic Environments}

Interestingly, when the environment is not economic, there can exist SCRs that are Nash implementable but not implementable by any $S$-oligarchic democracy. An example is provided next.

Example 4.7. Let $N=\{1,2,3,4\}, A=\{a, b\}$ and $\Theta=\left\{\theta, \theta^{\prime}, \theta^{\prime \prime}, \hat{\theta}, \hat{\theta}^{\prime}, \hat{\theta}^{\prime \prime}\right\}$. The preferences of the four players over alternatives in the six states are as follows:

$\begin{array}{cccccc}\theta & \theta^{\prime} & \theta^{\prime \prime} & \hat{\theta} & \hat{\theta}^{\prime} & \hat{\theta}^{\prime \prime} \\ a \succ_{1}^{\theta} b & a \succ_{1}^{\theta^{\prime}} b & b \sim_{1}^{\theta^{\prime \prime}} a & b \sim_{1}^{\hat{\theta}} a & a \succ_{1}^{\hat{\theta}^{\prime}} b & a \succ_{1}^{\hat{\theta}^{\prime \prime}} b \\ a \sim_{2}^{\theta} b & b \succ_{2}^{\theta^{\prime}} a & b \succ_{2}^{\theta^{\prime \prime}} a & b \succ_{2}^{\hat{\theta}} a & b \succ_{2}^{\hat{\theta}^{\prime}} a & a \sim_{2}^{\hat{\theta}^{\prime \prime}} b \\ a \sim_{3}^{\theta} b & b \succ_{3}^{\theta^{\prime}} a & b \succ_{3}^{\theta^{\prime \prime}} a & b \succ_{3}^{\hat{\theta}} a & a \sim_{3}^{\hat{\theta}^{\prime}} b & b \succ_{3}^{\hat{\theta}^{\prime \prime}} a \\ a \succ_{4}^{\theta} b & b \sim_{4}^{\theta^{\prime}} a & a \succ_{4}^{\theta^{\prime \prime}} b & b \sim_{4}^{\hat{\theta}} a & a \succ_{4}^{\hat{\theta}^{\prime}} b & a \succ_{4}^{\hat{\theta}^{\prime \prime}} b\end{array}$


This environment is not economic. For instance, in state $\theta, a \succeq_{i}^{\theta} b$ for all $i \in N$.

Let SCR $F$ be such that $F(\theta)=F\left(\hat{\theta}^{\prime}\right)=F\left(\hat{\theta}^{\prime \prime}\right)=\{a\}$ and $F\left(\theta^{\prime}\right)=F\left(\theta^{\prime \prime}\right)=F(\hat{\theta})=\{b\}$. $F$ is Maskin monotonic and hence, weak $S$-monotonic for any $S \subset N$. Furthermore, $F$ satisfies no-veto power. Hence, $F$ is Nash implementable.

We argue that $F$ is not implementable by $S$-oligarchic democracy for any $S \subset N$. Suppose there exists a sequence of $\left(S, \epsilon_{k}\right)$-oligarchic-democratic mechanisms $\left(\Gamma_{O\left(S, \epsilon_{k}\right)}\right)_{k=1}^{\infty}$ such that $\epsilon_{k} \in(0,1), \forall k$, with $\lim _{k \rightarrow \infty} \epsilon_{k} \rightarrow 0$, and for all $k,\left\{l\left[S, \epsilon_{k}, m\right]: m \in N E\left(\Gamma_{O\left(S, \epsilon_{k}\right)}, \tilde{\theta}\right)\right\}=F(\tilde{\theta})$ for all $\tilde{\theta} \in \Theta$.

Pick any $\epsilon_{k}$ and consider $m^{k} \in N E\left(\Gamma_{O\left(S, \epsilon_{k}\right)}, \theta^{\prime}\right)$. It must be that $g^{S}\left(m^{k S}\right)=g^{N}\left(m^{k}\right)=b$. Moreover, for any $m_{1} \in M_{1}$, we must have $g^{S}\left(\left(m_{1}, m_{-1}^{k}\right)^{S}\right)=g^{N}\left(m_{1}, m_{-1}^{k}\right)=b$ because otherwise, player 1 has an incentive to deviate to $m_{1}$ in state $\theta^{\prime}$. This further implies that there must exist $m_{4} \in M_{4}$ such that either $g^{S}\left(\left(m_{4}, m_{-4}^{k}\right)^{S}\right)=a$ or $g^{N}\left(m_{4}, m_{-4}^{k}\right)=a$ because otherwise, $m^{k} \in N E\left(\Gamma_{O\left(S, \epsilon_{k}\right)}, \theta\right)$.

If $4 \notin S$. Then $g^{S}\left(\left(m_{4}, m_{-4}^{k}\right)^{S}\right)=g^{S}\left(m^{k S}\right)=b$. Hence, it must be that $g^{N}\left(m_{4}, m_{-4}^{k}\right)=a$. We claim that $\left(m_{4}, m_{-4}^{k}\right) \in N E\left(\Gamma_{O\left(S, \epsilon_{k}\right)}, \theta\right)$. Clearly, players 2,3 and 4 do not have any improving unilateral deviations. On the other hand, if player 1 were to deviate to any $m_{1}$, then $g^{S}\left(\left(m_{1}, m_{4}, m_{-\{1,4\}}^{k}\right)^{S}\right)=g^{S}\left(\left(m_{1}, m_{-1}^{k}\right)^{S}\right)=b$. Hence, player 1 also does not have an improving unilateral deviation. Hence, $\left(m_{4}, m_{-4}^{k}\right) \in N E\left(\Gamma_{O\left(S, \epsilon_{k}\right)}, \theta\right)$. But then we have a contradiction since $g^{S}\left(\left(m_{4}, m_{-4}^{k}\right)^{S}\right)=b$. Therefore, $4 \in S$.

The above argument involved $\theta^{\prime}$ and $\theta$. Similarly, using $\theta^{\prime \prime}$ and $\theta$ we can argue that $1 \in S$; using $\hat{\theta}^{\prime}$ and $\hat{\theta}$ we can argue that $3 \in S$, and finally, using $\hat{\theta}^{\prime \prime}$ and $\hat{\theta}$ we can argue that $2 \in S$. Hence, we obtain a contradiction to the fact that $S \subset N$.

\subsubsection{Sufficient Conditions}

The environment in the previous example does not satisfy DTA- $S$ for all $S$. The problem for implementation using oligarchic democracy when $S$ is the set of oligarchs and the environment does not satisfy DTA- $S$ can be intuitively explained as follows. Suppose the players have expected-utility preferences. Let $a$ be in the SCR in state $\theta$ and consider the equilibrium $m$ of an $\left(S, \epsilon_{k}\right)$-oligarchic-democratic mechanism that implements $a$. It must be that by unilaterally changing her message, no oligarch can implement an alternative when the senate is $S$ that she strictly prefers to $a$ in state $\theta$. If this were not true, and if $\epsilon_{k}$ is small enough, the oligarch will prefer to deviate. Now, consider another state $\theta^{\prime}$ such that in going from state $\theta$ to $\theta^{\prime}$, alternative $a$ drops out of the SCR but for every oligarch, there is no reversal of her preferences over alternatives around $a$ (i.e., for all $i \in S, a \succeq_{i}^{\theta} b \Longrightarrow a \succeq_{i}^{\theta^{\prime}} b, \forall b$ ). Pick any message profile $m^{\prime}=\left(\left(m_{i}\right)_{i \in S},\left(m_{j}^{\prime}\right)_{j \notin S}\right)$, i.e., the messages of the oligarchs in $m^{\prime}$ are the 
same as in $m$. Since $m^{\prime S}=m^{S}$, alternative $a$ is still implemented when the senate is $S$. But $a$ is not in the SCR in state $\theta^{\prime}$ and hence, $m^{\prime}$ must not be an equilibrium in state $\theta^{\prime}$. Since for any oligarch, there is no reversal of her preferences over alternatives around $a$, it follows from the previous argument that no oligarch can implement a strictly preferred alternative when the senate is $S$. Thus, the only way an oligarch will deviate from $m^{\prime}$ is if she can implement a strictly preferred alternative when the senate is $N$. However, now suppose that $m^{\prime}$ is such that the alternative implemented when the senate is $N$ is unanimously most-preferred by all the players, which is possible since the environment it not economic. Then clearly, no player will have an incentive to deviate from $m^{\prime}$ and hence, we will not be able to implement the SCR.

As the next result shows, this problem can be avoided if we restrict attention to environments satisfying DTA-S (proof is in the Appendix).

Theorem 4.8. Suppose $3 \leq|S|<n$ and $\mathcal{E}$ satisfies DTA-S. Any Maskin monotonic SCR $F$ that satisfies no-veto power is implementable by $S$-oligarchic democracy.

Stronger results can be established under further restrictions on the environments. For instance, consider the class of environments that satisfy both DTA- $S$ and unique-top condition. Note that this class of environments is not a subset of economic environments. The next result shows that we can drop the requirement of no-veto power in this class of environments (proof is in the Appendix).

Theorem 4.9. Suppose $3 \leq|S|<n$ and $\mathcal{E}$ satisfies DTA-S and unique-top condition. Any Maskin monotonic SCR F is implementable by S-oligarchic democracy.

As an application of the above theorem, consider an environment with single-peaked preferences. For instance, suppose a large society faces the problem of implementing a tax rate. A state describes the "ideological" biases of each individual. The "left-wing" individuals have the peaks of their preferences at higher tax rates whereas "right-wing" individuals have the peaks of their preferences at lower tax rates. Furthermore, suppose that there are two individuals, say $i_{l}$ and $i_{r}$, whose "ideological" biases never coincide, i.e., the peaks of their preferences are different in every state. Then the above theorem says that we can implement any Maskin monotonic SCR by oligarchic democracy if we designate individuals $i_{l}, i_{r}$ and any other third individual $j$ as oligarchs.

Remark 4.10. We are unable to strengthen either Theorem 4.8 or 4.9 by replacing Maskin monotonicity with weak $S$-monotonicity of the SCR. However, we can reduce this gap between necessary and sufficient conditions in case of environments satisfying unique-top condition. That is, suppose $3 \leq|S|<n$ and $\mathcal{E}$ satisfies DTA-S and unique-top condition. Then 
any weak $S$-monotonic SCR $F$ that satisfies no-veto power is implementable by $S$-oligarchic democracy (proof upon request).

\section{Implementation by Random Sampling}

\subsection{Necessary Condition}

We first present the necessary condition. Recall the definitions of $\mathcal{N}_{\bar{n}}$ and let $\mathcal{N}_{\bar{n}}(i)$ be the set of all senates $S \in \mathcal{N}_{\bar{n}}$ such that $i \in S$. For any function $h: \mathcal{N}_{\bar{n}} \rightarrow A$, define the lottery induced by $h$, denoted by $l[h]$, as the lottery that assigns to each $a \in A$ the probability $\left|\left\{S \in \mathcal{N}_{\bar{n}}: h(S)=a\right\}\right| /\left|\mathcal{N}_{\bar{n}}\right|$.

Definition 5.1. SCR $F$ is $R(\bar{n})$-monotonic if whenever $a \in F(\theta)$ and $a \notin F(\theta)$ for some $a$, $\theta$ and $\theta^{\prime}$, then there exist player $i \in N$ and function $h_{i}: \mathcal{N}_{\bar{n}} \rightarrow A$ such that

$$
h_{i}(S)=a, \forall S \in \mathcal{N}_{\bar{n}} \backslash \mathcal{N}_{\bar{n}}(i), \quad a \succeq_{i}^{\theta} l\left[h_{i}\right] \text { and } l\left[h_{i}\right] \succ_{i}^{\theta^{\prime}} a .
$$

Thus, $R(\bar{n})$-monotonicity requires that whenever an alternative $a$ is in the SCR for some state $\theta$ but drops out of the SCR in state $\theta^{\prime}$, then in going from $\theta$ to $\theta^{\prime}$, there must exist some player $i$ for whom there is reversal of her preferences over lotteries around $a$ (i.e., there is a lottery that she weakly prefers to $a$ in $\theta$ but this is not true in $\theta^{\prime}$ ), where we consider only those lotteries that are induced by all possible mappings $h_{i}: \mathcal{N}_{\bar{n}} \rightarrow A$ with the property that $h_{i}(S)=a$ for any $S \in \mathcal{N}_{\bar{n}} \backslash \mathcal{N}_{\bar{n}}(i)$.

The following theorem shows that $R(\bar{n})$-monotonicity is necessary for implementation by $\bar{n}$-random sampling.

Theorem 5.2. If SCR $F$ is implementable by $\bar{n}$-random sampling, then $F$ is $R(\bar{n})$-monotonic.

Proof. Let $\Gamma=\left(\left(M_{i}\right)_{i \in N}, R(\bar{n}),\left(g^{S}\right)_{S \in \mathcal{N}}\right)$ be the $R(\bar{n})$-sampling mechanism that implements $F$. Suppose there exist $a, \theta$ and $\theta^{\prime}$ such that $a \in F(\theta)$ but $a \notin F\left(\theta^{\prime}\right)$. Then there must exist $m \in N E\left(\Gamma_{R(\bar{n})}, \theta\right)$ with $l[R(\bar{n}), m]=a$, i.e., $g^{S}\left(m^{S}\right)=a$ for all $S \in \mathcal{N}_{\bar{n}}$. Since $a \notin F\left(\theta^{\prime}\right)$, we have $m \notin N E\left(\Gamma_{R(\bar{n})}, \theta^{\prime}\right)$. Hence, there must exist $i \in N$ and $m_{i}^{\prime} \in M_{i}$ such that

$$
a \succeq_{i}^{\theta} l\left[R(\bar{n}),\left(m_{i}^{\prime}, m_{-i}\right)\right] \text { and } l\left[R(\bar{n}),\left(m_{i}^{\prime}, m_{-i}\right)\right] \succ_{i}^{\theta^{\prime}} a
$$

Lottery $l\left[R(\bar{n}),\left(m_{i}^{\prime}, m_{-i}\right)\right]$ assigns to each alternative $a^{\prime} \in A$, the probability of $\mid\left\{S \in \mathcal{N}_{\bar{n}}\right.$ : $\left.g^{S}\left(\left(m_{i}^{\prime}, m_{-i}\right)^{S}\right)=a^{\prime}\right\}|/| \mathcal{N}_{\bar{n}} \mid$. But $g^{S}\left(\left(m_{i}^{\prime}, m_{-i}\right)^{S}\right)=g^{S}\left(m^{S}\right)=a$ for all $S \in \mathcal{N}_{\bar{n}} \backslash \mathcal{N}_{\bar{n}}(i)$. Hence, player $i$ and function $h_{i}: \mathcal{N}_{\bar{n}} \rightarrow A$ such that $h_{i}(S)=g^{S}\left(\left(m_{i}^{\prime}, m_{-i}\right)^{S}\right)$ for all $S \in \mathcal{N}_{\bar{n}}$ satisfy the required condition. 
The proof clarifies why $R(\bar{n})$-monotonicity requires the particular reversal of preferences over lotteries. In $\bar{n}$-random sampling, the messages of any player $i$ are considered only if she is selected in the sample, i.e., whenever the senate is $S \in \mathcal{N}_{\bar{n}}(i)$. Hence, by changing her message, player $i$ can change the alternative that is implemented when the senate is $S$ only if $S \in \mathcal{N}_{\bar{n}}(i)$. That is why, whenever player $i$ has an improving unilateral deviation in $R(\bar{n})$ sampling mechanism, it must be that she is able to generate a strictly preferred lottery that is induced by some $h_{i}: \mathcal{N}_{\bar{n}} \rightarrow A$ with the property that $h_{i}(S)=a$ for any $S \in \mathcal{N}_{\bar{n}} \backslash \mathcal{N}_{\bar{n}}(i)$.

The particular reversal of preferences over lotteries around an alternative required in $R(\bar{n})$-monotonicity is obviously weaker than the reversal of preferences over alternatives around an alternative required in Maskin monotonicity. Hence, we easily have the following lemma (proof is omitted):

Lemma 5.3. If $F$ is Maskin monotonic, then $F$ is $R(\bar{n})$-monotonic for all positive $\bar{n}<n$.

\subsection{Sufficient Condition}

The next result gives the sufficient condition for implementation by $\bar{n}$-random sampling.

Theorem 5.4. Let $4 \leq \bar{n}<n$ and $\mathcal{E}$ satisfy $D T A-N$ and top-coincidence condition. If $S C R$ $F$ is Maskin monotonic, then $F$ is implementable by $\bar{n}$-random sampling.

Sketch of the proof: For each player $j \in N$, let

$$
\mathbb{S}(j)= \begin{cases}\left\{S \in \mathcal{N}_{\bar{n}}(j):\{1,2\} \subset S\right\}, & \text { if } j>2 . \\ \left\{S \in \mathcal{N}_{\bar{n}}(j):\{3,4\} \subset S\right\}, & \text { if } j \in\{1,2\} .\end{cases}
$$

Define the $R(\bar{n})$-sampling mechanism $\Gamma_{R(\bar{n})}=\left(\left(M_{i}\right)_{i \in N}, R(\bar{n}),\left(g^{S}\right)_{S \in \mathcal{N}}\right)$ in which for all $i \in N$,

$$
M_{i}=\Theta \times A \times A \times \mathbb{Z}_{+} .
$$

Let a typical message $m_{i}$ be of the form $\left(\theta_{i}, a_{i}^{1}, a_{i}^{2}, z_{i}\right)$.

For each $S \in \mathcal{N}_{\bar{n}}$, the outcome function $g^{S}$ is as follows:

(i) If for every player $i \in S, m_{i}=\left(\theta, a_{i}^{1}, a, 0\right)$ and $a \in F(\theta)$, then $g^{S}\left(\left(m_{i}\right)_{i \in S}\right)=a$

(ii) If for $|S|-1$ players $i \neq j$ in $S, m_{i}=\left(\theta, a_{i}^{1}, a, 0\right)$ and $a \in F(\theta)$, but $m_{j}=\left(\theta_{j}, a_{j}^{1}, a_{j}^{2}, z_{j}\right) \neq$ $\left(\theta, a_{j}^{1}, a, 0\right)$, then

$$
g^{S}\left(\left(m_{i}\right)_{i \in S}\right)=\left\{\begin{array}{cl}
a & \text { if } a_{j}^{2} \succ_{j}^{\theta} a \text { or } S \notin \mathbb{S}(j) \\
a_{j}^{2} & \text { if } a \succeq_{j}^{\theta} a_{j}^{2} \text { and } S \in \mathbb{S}(j) .
\end{array}\right.
$$


(iii) In all other cases, $g^{S}\left(\left(m_{i}\right)_{i \in S}\right)=a_{j}^{1}$ where $j \in S$ is the player with the lowest index among those players in $S$ who announce the highest integer in the profile $\left(m_{i}\right)_{i \in S}$.

Finally, for all $S^{\prime} \in \mathcal{N} \backslash \mathcal{N}_{\bar{n}}$, the outcome function $g^{S^{\prime}}$ can be arbitrary.

Thus, each player sends a message with four components $\left(\theta_{i}, a_{i}^{1}, a_{i}^{2}, z_{i}\right)$. For every possible senate $S \in \mathcal{N}_{\bar{n}}$ that can be selected by random sampling, the outcome function $g^{S}$ is defined like in the canonical mechanism of Maskin (1999) with three rules. The first rule is used when there is agreement in all the messages sampled by the planner, except possibly in the second components $a_{i}^{1}$. In this case, the planner implements the commonly agreed alternative. The second rule allows the planner to use Maskin monotonicity of the SCR to eliminate the possibility that players agree on a message with a "bad" alternative. In such a situation, rule (ii) gives any player $j$ the opportunity to deviate and implement a strictly preferred alternative $a_{j}^{2}$ if the senate is $S \in \mathbb{S}(j)$ by using the reversal of her preferences over alternatives around the "bad" alternative. However, if the senate is $S \notin \mathbb{S}(j)$, then player $j$ cannot undo the implementation of the "bad" alternative by being the only one to disagree with the rest of the players in $S$. Since the probability that some $S \in \mathbb{S}(j)$ will be sampled is positive, the probability that $a_{j}^{2}$ will be implemented is positive. Moreover, deviations by player $j$ do not affect the alternative implemented when the sampled senate is $S \in \mathcal{N}_{\bar{n}} \backslash \mathbb{S}(j)$. This provides sufficient incentives for $j$ to deviate. Finally, the third rule eliminates the possibility of any equilibria other than those in which all players agree in their messages. There are two possibilities:

(a) There are at least three individuals whose messages disagree amongst each other. In this case, we find an alternative that is unanimously most-preferred by all players, which contradicts DTA- $N$. To see how this is done, suppose one of these three players is player $i_{1}$ who is defined as the player with the lowest index among the players in $N$ who announce the highest integer (the proof takes care of other cases). Then for any player $j$, we consider the senate in which the three disagreeing players are selected along with player $j$, which is possible since the sample size is four. Due to the disagreement, rule (iii) is used in this senate, and hence alternative announced by $i_{1}$ in the second component of her message, say $a_{i_{1}}^{1}$, is implemented. Since rule (iii) is used in this senate, player $j$ could have also announced her most-preferred alternative and a higher integer than that of $i_{1}$ in order to implement her most-preferred alternative in this senate. Furthermore, since rule (ii) does not depend on the second and last components of player $j$ 's message, she would not be worse-off by this deviation in any other senate selection. Therefore, it must be that $a_{i}^{1}$ is most-preferred by all players $j$, contradicting DTA- $N$.

(b) There are only two individuals whose messages disagree (as already pointed out, the 
cases when all players agree are taken care of by rules (i) and (ii)). Then every other player agrees with at least one of these two and hence, there is a unique disagreeing player, say $i$. In this case, we find two alternatives that are most-preferred by $n-1$ players, which contradicts top-coincidence condition. To do this, for any player $j \neq i$, we consider two senates that contain both players $j$ and $i$ such that one senate $S_{1}^{\prime}$ is in $\mathbb{S}(i)$ and the other senate $S_{2}^{\prime}$ is not in $\mathbb{S}(i)$. This is possible because $\bar{n} \geq 4$. Due to the presence of a unique disagreeing player, rule (ii) is used in both these senates. Suppose the alternative announced by $j$ in her third component, say $a^{2}$ - which is the same for all $j \neq i$ since their messages coincide - , is weakly preferred by $i$ to her third component, say $a_{i}^{2}$, and $a^{2} \neq a_{i}^{2}$ (the proof takes care of other cases). Then $a_{i}^{2}$ is implemented if the senate is $S_{1}^{\prime}$ and $a^{2}$ is implemented if the senate is $S_{2}^{\prime}$. Now, if either $a^{2}$ or $a_{i}^{2}$ is not a most-preferred alternative of $j$, then player $j$ can be better-off by deviating like in (a) above. Thus, we obtain that both $a^{2}$ and $a_{i}^{2}$ must be most-preferred by all player $j \neq i$, contradicting top-coincidence condition.

The above arguments are formalized in the proof presented in the Appendix.

Since every economic environment satisfies both DTA- $N$ and top-coincidence condition, we have the following corollary:

Corollary 5.5. Let $4 \leq \bar{n}<n$ and $\mathcal{E}$ be an economic environment. If $S C R F$ is Maskin monotonic, then $F$ is implementable by $\bar{n}$-random sampling.

Remark 5.6. We are unable to strengthen either Theorem 5.4 or Corollary 5.5 by replacing Maskin monotonicity with $R(\bar{n})$-monotonicity of the SCR. However, we can close this gap between necessary and sufficient conditions in case of economic environments that satisfy unique-top condition. That is, let $4 \leq \bar{n}<n$ and $\mathcal{E}$ be an economic environment satisfying unique-top condition. Then any $R(\bar{n})$-monotonic SCR $F$ is implementable by $\bar{n}$-random sampling (proof upon request).

\subsection{Comparison}

The following result easily follows from Theorem 2.9 and Corollary 5.5:

Corollary 5.7. Suppose $4 \leq \bar{n}<n$ and $\mathcal{E}$ is an economic environment. If $F$ is Nash implementable, then $F$ is implementable by $\bar{n}$-random sampling.

Thus, in economic environments, any SCR that is implementable by direct democracy (i.e., Nash implementable) is also implementable by randomly sampling only four - or more - messages of the players.

The next example shows that even in economic environments, four is the minimal sample size that guarantees the implementation of Maskin monotonic SCRs by random sampling. 
Example 5.8. Let $N=\{1,2,3,4,5\}, A=\{a, b\}$ and $\Theta=\left\{\theta, \theta^{\prime}\right\}$. The preferences of the five players over alternatives in the two states are as follows:

$\begin{array}{cc}\theta & \theta^{\prime} \\ a \succ_{1}^{\theta} b & a \succ_{1}^{\theta^{\prime}} b \\ a \succ_{2}^{\theta} b & a \succ_{2}^{\theta^{\prime}} b \\ a \succ_{3}^{\theta} b & b \succ_{3}^{\theta^{\prime}} a \\ b \succ_{4}^{\theta} a & b \succ_{4}^{\theta^{\prime}} a \\ b \succ_{5}^{\theta} a & b \succ_{5}^{\theta^{\prime}} a\end{array}$

Let SCR $F$ be such that $F(\theta)=\{a, b\}$ and $F\left(\theta^{\prime}\right)=\{b\}$. The environment is economic and $F$ is Maskin monotonic. Hence, $F$ is Nash implementable.

We argue that $F$ is not implementable by $R(\bar{n})$-random sampling for any $\bar{n} \leq 3$. Suppose there exists a $R(\bar{n})$-sampling mechanism $\Gamma_{R(\bar{n})}$ such that $\left\{l[R(\bar{n}), m]: m \in N E\left(\Gamma_{R(\bar{n})}, \tilde{\theta}\right)\right\}=$ $F(\tilde{\theta})$ for all $\tilde{\theta} \in \Theta$.

Consider $m \in N E\left(\Gamma_{R(\bar{n})}, \theta\right)$ such that $l[R(\bar{n}), m]=a$. It must be that $g^{S}\left(m^{S}\right)=a, \forall S \in$ $\mathcal{N}_{\bar{n}}$. Moreover, for $i \in\{4,5\}$, there must not exist any $m_{i}^{\prime}$ such that for some $S \in \mathcal{N}_{\bar{n}}$ with $i \in S$, we have $g^{S}\left(\left(m_{i}^{\prime}, m_{-i}\right)^{S}\right)=b$; otherwise, player $i$ has an incentive to deviate to $m_{i}^{\prime}$ in state $\theta$. Consider $\hat{m} \in N E\left(\Gamma_{R(\bar{n})}, \theta\right)$ such that $l[R(\bar{n}), \hat{m}]=b$. It must be that $g^{S}\left(\hat{m}^{S}\right)=b, \forall S \in \mathcal{N}_{\bar{n}}$. Moreover, for $i \in\{1,2,3\}$, there must not exist any $m_{i}^{\prime}$ such that for some $S \in \mathcal{N}_{\bar{n}}$ with $i \in S$, we have $g^{S}\left(\left(m_{i}^{\prime}, \hat{m}_{-i}\right)^{S}\right)=a$; otherwise, player $i$ has an incentive to deviate to $m_{i}^{\prime}$ in state $\theta$.

Consider the message profile $\tilde{m}=\left(m_{1}, m_{2}, m_{3}, \hat{m}_{4}, \hat{m}_{5}\right)$.

- Suppose $\bar{n}=3$. If $S \in\{1,4,5\} \bigcup\{2,4,5\} \bigcup\{3,4,5\}$, then $g^{S}\left(\tilde{m}^{S}\right)=b$ whereas if $S \in \mathcal{N}_{\bar{n}} \backslash\{1,4,5\} \bigcup\{2,4,5\} \bigcup\{3,4,5\}$, then $g^{S}\left(\hat{m}^{S}\right)=a$. Then $\tilde{m} \in N E\left(\Gamma_{R(\bar{n})}, \theta\right)$ since players 1, 2 and 3 cannot unilaterally change the alternative implemented for any $S \in\{1,4,5\} \bigcup\{2,4,5\} \bigcup\{3,4,5\}$ whereas players 4 and 5 cannot unilaterally change the alternative implemented for any $S \in \mathcal{N}_{\bar{n}} \backslash\{1,4,5\} \bigcup\{2,4,5\} \bigcup\{3,4,5\}$. But both $a$ and $b$ are in the support of the lottery $l[R(\bar{n}), \tilde{m}]$, a contradiction.

- Suppose $\bar{n}=2$. Then for $S=\{3,4\}$, we have $g^{S}\left(\tilde{m}^{S}\right)=g^{S}\left(m_{3}, \hat{m}_{4}\right)$. We have already argued that for $S=\{3,4\}$, there does not exist any $m_{4}^{\prime}$ such that $g^{S}\left(m_{3}, m_{4}^{\prime}\right)=b$. Hence, $g^{S}\left(m_{3}, \hat{m}_{4}\right)=a$. For $S=\{3,4\}$, we have also argued that there does not exist any $m_{3}^{\prime}$ such that $g^{S}\left(m_{3}^{\prime}, \hat{m}_{4}\right)=a$. Hence, $g^{S}\left(m_{3}, \hat{m}_{4}\right)=b$, a contradiction.

- Suppose $\bar{n}=1$. We have already argued that for $S=\{4\}$, there does not exist any $m_{4}^{\prime}$ such that $g^{S}\left(\left(m_{4}^{\prime}, m_{-4}\right)^{S}\right)=g^{S}\left(m_{4}^{\prime}\right)=b$. But that contradicts the fact that 


$$
g^{S}\left(\hat{m}_{4}\right)=b .
$$

The next two examples show that the sufficiency result of Theorem 5.4 is not longer true if we consider environments outside the class of environments satisfying DTA- $N$ and top-coincidence condition. In the first example, the environment satisfies top-coincidence condition but not DTA- $N$. The SCR in the example is Nash implementable as it satisfies Maskin monotonicity and no-veto power but it is not implementable by $\bar{n}$-random sampling when sample size $\bar{n}=4$.

Example 5.9. Let $N=\{1,2,3,4,5\}, A=\{a, b\}$ and $\Theta=\left\{\theta, \theta^{\prime}\right\}$. The preferences of the five players over alternatives in the two states are as follows:

$\begin{array}{cc}\theta & \theta^{\prime} \\ a \sim_{1}^{\theta} b & b \succ_{1}^{\theta^{\prime}} a \\ a \sim_{2}^{\theta} b & b \succ_{2}^{\theta^{\prime}} a \\ a \sim_{3}^{\theta} b & b \succ_{3}^{\theta^{\prime}} a \\ a \succ_{4}^{\theta} b & a \succ_{4}^{\theta^{\prime}} b \\ a \succ_{5}^{\theta} b & b \succ_{5}^{\theta^{\prime}} a\end{array}$

Let SCR $F$ be such that $F(\theta)=\{a\}$ and $F\left(\theta^{\prime}\right)=\{b\}$. Since $F$ is Maskin monotonic and satisfies no-veto power, $F$ is Nash implementable.

The environment satisfies top-coincidence condition but does not satisfy DTA- $N$ since $a$ is the unanimously most-preferred alternative in state $\theta$. We argue that $F$ is not implementable by $R(\bar{n})$-random sampling for $\bar{n}=4$. Suppose there exists a $R(\bar{n})$-sampling mechanism $\Gamma_{R(\bar{n})}$ such that $\left\{l[R(\bar{n}), m]: m \in N E\left(\Gamma_{R(\bar{n})}, \tilde{\theta}\right)\right\}=F(\tilde{\theta})$ for all $\tilde{\theta} \in \Theta$.

Consider $m \in N E\left(\Gamma_{R(\bar{n})}, \theta^{\prime}\right)$ such that $l[R(\bar{n}), m]=b$. It must be that $g^{S}\left(m^{S}\right)=b, \forall S \in$ $\mathcal{N}_{\bar{n}}$. Moreover, there must not exist any $m_{4}^{\prime}$ such that for some $S \in \mathcal{N}_{\bar{n}}$ with $4 \in S$, we have $g^{S}\left(\left(m_{4}^{\prime}, m_{-4}\right)^{S}\right)=a$; otherwise, player 4 has an incentive to deviate to $m_{4}^{\prime}$ in state $\theta^{\prime}$. Since $b \notin F(\theta)$, it must be that $m \notin N E\left(\Gamma_{R(\bar{n})}, \theta\right)$. Now, only player $i \in\{4,5\}$ is such that there exists an alternative that she prefers to $b$ in state $\theta$. However, given $m_{-4}$, player 4 cannot change the alternative implemented for any senate by changing her message. Hence, it must be player 5 for whom there exists $m_{5}^{\prime}$ such that for some $S \in \mathcal{N}_{\bar{n}}$ with $5 \in S$, we have $g^{S}\left(\left(m_{5}^{\prime}, m_{-5}\right)^{S}\right)=a$. Without loss of generality, let $m_{5}^{\prime}$ be the best response to $m_{-5}$ in state $\theta$.

If $m^{\prime}=\left(m_{5}^{\prime}, m_{-5}\right) \in N E\left(\Gamma_{R(\bar{n})}, \theta\right)$, then alternative $b$ is implemented when the senate is $S=\{1,2,3,4\}$, a contradiction. Hence, $m^{\prime} \notin N E\left(\Gamma_{R(\bar{n})}, \theta\right)$. Since $m_{5}^{\prime}$ is a best response to $m_{-5}=\left(m_{1}, m_{2}, m_{3}, m_{4}\right)$, and players 1,2 , and 3 are indifferent between $a$ and $b$, it must be that player 4 has an improving unilateral deviation. Let $\hat{m}_{4}$ be the best response to $m_{-4}^{\prime}=$ 
$\left(m_{1}, m_{2}, m_{3}, m_{5}^{\prime}\right)$. Since $m^{\prime}$ was such that for at least one $S \in \mathcal{N}_{\bar{n}}$, we have $g^{S}\left(m^{\prime S}\right)=a$, the new message profile $\hat{m}=\left(m_{1}, m_{2}, m_{3}, \hat{m}_{4}, m_{5}^{\prime}\right)$ must be such that for at least two $S \in \mathcal{N}_{\bar{n}}$, we have $g^{S}\left(\hat{m}^{S}\right)=a$; otherwise, player 4 will not strictly improve with her deviation to $\hat{m}_{4}$. If $\hat{m} \in N E\left(\Gamma_{R(\bar{n})}, \theta\right)$, then the alternative $b$ is implemented when the senate is $S=\{1,2,3,4\}$ because, as already argued, $g^{S}\left(m_{1}, m_{2}, m_{3}, \hat{m}_{4}\right)=b$, which is a contradiction. Hence, $\hat{m} \notin$ $N E\left(\Gamma_{R(\bar{n})}, \theta\right)$. Since $\hat{m}_{4}$ is a best response to $\hat{m}_{-4}=\left(m_{1}, m_{2}, m_{3}, m_{5}^{\prime}\right)$, and players 1,2 , and 3 are indifferent between $a$ and $b$, it must be that player 5 has an improving unilateral deviation. Let $\tilde{m}_{5}$ be the best response to $\hat{m}_{-5}=\left(m_{1}, m_{2}, m_{3}, \hat{m}_{4}\right)$. Since $\hat{m}$ was such that for at least two $S \in \mathcal{N}_{\bar{n}}$, we have $g^{S}\left(\hat{m}^{S}\right)=a$, the new message profile $\tilde{m}=\left(m_{1}, m_{2}, m_{3}, \hat{m}_{4}, \tilde{m}_{5}\right)$ must be such that for at least three $S \in \mathcal{N}_{\bar{n}}$, we have $g^{S}\left(\tilde{m}^{S}\right)=a$; otherwise, player 5 will not strictly improve with her deviation to $\tilde{m}_{5}$. If $\tilde{m} \in N E\left(\Gamma_{R(\bar{n})}, \theta\right)$, then the alternative $b$ is implemented when the senate is $S=\{1,2,3,4\}$ because $g^{S}\left(m_{1}, m_{2}, m_{3}, \hat{m}_{4}\right)=b$, which is a contradiction. Hence, $\tilde{m} \notin N E\left(\Gamma_{R(\bar{n})}, \theta\right)$. By repeating the above argument, player 4 will switch to her best response $\hat{m}_{4}^{\prime}$ and the message profile $\hat{m}^{\prime}=\left(m_{1}, m_{2}, m_{3}, \hat{m}_{4}^{\prime}, \tilde{m}_{5}\right)$ will be such that for at least four $S \in \mathcal{N}_{\bar{n}}$, we have $g^{S}\left(\hat{m}^{\prime S}\right)=a$. This means that for all $S \in N_{\bar{n}}$ such that $5 \in S$, we have $g^{S}\left(\hat{m}^{\prime S}\right)=a$. Then $\hat{m}^{\prime} \in N E\left(\Gamma_{R(\bar{n})}, \theta\right)$ because player 4 is already playing a best response to $\tilde{m}_{-4}=\left(m_{1}, m_{2}, m_{3}, \tilde{m}_{5}\right)$ and player 5 cannot change the alternative implemented when $S=\{1,2,3,4\}$. However, $g^{S}\left(m_{1}, m_{2}, m_{3}, \hat{m}_{4}^{\prime}\right)=b$ for $S=\{1,2,3,4\}$, a contradiction.

In the next example, the environment satisfies DTA- $N$ but not top-coincidence condition. The SCR is Nash implementable but it is not implementable by $\bar{n}$-random sampling when sample size $\bar{n}=4$.

Example 5.10. Let $N=\{1,2,3,4,5\}, A=\{a, b, c\}$ and $\Theta=\left\{\theta, \theta^{\prime}\right\}$. The preferences of the five players over alternatives in the two states are as follows:

$\begin{array}{cc}\theta & \theta^{\prime} \\ c \succ_{1}^{\theta} a \sim_{1}^{\theta} b & c \succ_{1}^{\theta^{\prime}} a \succ_{1}^{\theta^{\prime}} b \\ a \sim_{2}^{\theta} b \succ_{2}^{\theta} c & a \sim_{2}^{\theta^{\prime}} b \succ_{2}^{\theta^{\prime}} c \\ a \sim_{3}^{\theta} b \succ_{3}^{\theta} c & a \sim_{3}^{\theta^{\prime}} b \succ_{3}^{\theta^{\prime}} c \\ a \sim_{4}^{\theta} b \succ_{4}^{\theta} c & a \sim_{4}^{\theta^{\prime}} b \succ_{4}^{\theta^{\prime}} c \\ a \sim_{5}^{\theta} b \succ_{5}^{\theta} c & a \sim_{5}^{\theta^{\prime}} b \succ_{5}^{\theta^{\prime}} c\end{array}$

Let SCR $F$ be such that $F(\theta)=\{a, b\}$ and $F\left(\theta^{\prime}\right)=\{a\} . \quad F$ is Maskin monotonic. However, $F$ does not satisfy no-veto power since in $\theta^{\prime}, b$ is most-preferred alternative for four players but $b \notin F\left(\theta^{\prime}\right)$. Still, $F$ is Nash implementable. To show this, first we argue that $F$ is implementable by $S$ oligarchy, where $S=\{1\}$. Define an $S$-oligarchic mechanism such 
that $M_{1}=\{a, b\}$ and $g^{S}\left(m_{1}\right)=m_{1}$ for all $m_{1} \in M_{1}$. Since only the message of player 1 is transmitted to the planner, in state $\theta$, player 1 is indifferent between announcing messages $a$ and $b$ whereas in state $\theta^{\prime}$, player 1 will announce $a$. Thus, $\left\{g^{S}\left(m^{S}\right): m \in N E\left(\Gamma_{O(S)}, \theta\right)\right\}=$ $\{a, b\}$ and $\left\{g^{S}\left(m^{S}\right): m \in N E\left(\Gamma_{O(S)}, \theta^{\prime}\right)\right\}=\{a\}$. Hence, $F$ is implementable by $S=\{1\}$ oligarchy. It follows from Proposition 3.6 that $F$ is Nash implementable.

The environment satisfies DTA- $N$ but not top-coincidence condition since both $a$ and $b$ are most-preferred alternatives for all players 2 through 5 in both states $\theta$ and $\theta^{\prime}$. We argue that $F$ is not implementable by $R(\bar{n})$-random sampling for $\bar{n}=4$. Suppose there exists a $R(\bar{n})$-sampling mechanism $\Gamma_{R(\bar{n})}$ such that $\left\{l[R(\bar{n}), m]: m \in N E\left(\Gamma_{R(\bar{n})}, \tilde{\theta}\right)\right\}=F(\tilde{\theta})$ for all $\tilde{\theta} \in \Theta$.

Consider $m \in N E\left(\Gamma_{R(\bar{n})}, \theta\right)$ such that $l[R(\bar{n}), m]=b$. It must be that $g^{S}\left(m^{S}\right)=b, \forall S \in$ $\mathcal{N}_{\bar{n}}$. Observe that for any $m_{1}^{\prime \prime}$ and $S \in \mathcal{N}_{\bar{n}}$ which includes player $1, g^{S}\left(\left(m_{1}^{\prime \prime}, m_{-1}\right)^{S}\right) \neq c$; otherwise, player 1 would unilaterally deviate to $m_{1}^{\prime \prime}$ from $m$ in state $\theta$. Moreover, because $b \notin F\left(\theta^{\prime}\right)$, it must be that $b \notin N E\left(\Gamma_{R(\bar{n})}, \theta^{\prime}\right)$. Hence, there must exist player $i, m_{i}^{\prime} \in M_{i}$ and $S \in \mathcal{N}_{\bar{n}}$ such that $g^{S}\left(\left(m_{i}^{\prime}, m_{-i}\right)^{S}\right) \succ_{i}^{\theta^{\prime}} b$. Because $b$ is a most-preferred alternative for players 2 through 5 in state $\theta^{\prime}$, it must be that $i=1$. We already know $g^{S}\left(\left(m_{1}^{\prime}, m_{-1}\right)^{S}\right) \neq c$. Consequently, $g^{S}\left(\left(m_{1}^{\prime}, m_{-1}\right)^{S}\right)=a$.

Now consider $\left(m_{1}^{\prime}, m_{-1}\right)$. As argued, alternative $a$ is implemented for some $S \in \mathcal{N}_{\bar{n}}$ such that $1 \in S$. However, alternative $b$ is implemented when the senate is $S=\{2,3,4,5\}$ since $g^{S}\left(\left(m_{1}^{\prime}, m_{-1}\right)^{S}\right)=g^{S}\left(m^{S}\right)=b$ as $\left(m_{1}^{\prime}, m_{-1}\right)^{S}=m^{S}$. Hence, to reach the desired contradiction, it suffices to show $\left(m_{1}^{\prime}, m_{-1}\right) \in N E\left(\Gamma_{R(\bar{n})}, \theta\right)$.

If $1 \notin S \in \mathcal{N}_{\bar{n}}$, then $g^{S}\left(\left(m_{1}^{\prime}, m_{-1}\right)^{S}\right)=b$ because $\left(m_{1}^{\prime}, m_{-1}\right)^{S}=m^{S}$. In addition, if $1 \in S \in \mathcal{N}_{\bar{n}}$, then $g^{S}\left(\left(m_{1}^{\prime}, m_{-1}\right)^{S}\right)$ is either $a$ or $b$. Since both alternatives $a$ and $b$ are most-preferred by players 2 through 5 in state $\theta$, none of these players has an incentive to deviate from $\left(m_{1}^{\prime}, m_{-1}\right)$. We already know that for any $m_{1}^{\prime \prime}$ and $S \in \mathcal{N}_{\bar{n}}$ which includes player $1, g^{S}\left(\left(m_{1}^{\prime \prime}, m_{-1}\right)^{S}\right) \neq c$. Therefore, for any unilateral deviation by player 1 from $\left(m_{1}^{\prime}, m_{-1}\right)$, either $a$ or $b$ is implemented. But player 1 is indifferent between $a$ and $b$ in state $\theta$. Hence, player 1 also has no incentive to deviate from $\left(m_{1}^{\prime}, m_{-1}\right)$ in state $\theta$. Thus, $\left(m_{1}^{\prime}, m_{-1}\right) \in N E\left(\Gamma_{R(\bar{n})}, \theta\right)$.

\section{Conclusion}

Within the large class of economic environments, our results suggest that costs and time involved in collecting opinions of individuals in a community in order implement Maskin monotonic SCRs can be substantially reduced by using alternative political processes. For simplicity, let's assume that there is a fixed cost $c$ of collecting each individual's message. 
Then the cost incurred in direct democracy is $n c$. On the other hand, our result on oligarchic democracy implies that the expected cost of collecting individuals' messages can be reduced to arbitrarily close to $3 c$ in economic environments. However, there is a positive, though very small chance, that the realized cost will be $n c$ when $N$ is selected as the senate in oligarchic democracy. If we are concerned with the ex-post cost of collecting individuals' messages, then random sampling with sample size of four can be used in economic environments, guaranteeing an ex-post cost of only $4 c$.

Our positive results, however, need not carry over to incomplete information environments. For instance, if players have private values, then the state of the world cannot be known even if $n-1$ individuals truthfully report their types. Thus, SCRs that are not "measurable" with respect to the information of the selected subset of individuals cannot be implemented in such an environment. Nevertheless, we expect that similar positive results can be obtained for incomplete information environments in which some subsets of the individuals are "better" informed than others. We leave these issues for future research.

\section{Appendix}

Proof of Theorem 4.4: Consider the sequence of $\left(S, \epsilon_{k}\right)$-oligarchic-democratic mechanisms $\left(\Gamma_{O\left(S, \epsilon_{k}\right)}\right)_{k=1}^{\infty}$ defined in the sketch of the proof.

Step 1. For all $k$ and $\theta \in \Theta, F(\theta) \subseteq\left\{l\left[S, \epsilon_{k}, m\right]: m \in N E\left(\Gamma_{O\left(S, \epsilon_{k}\right)}, \theta\right)\right\}$.

Fix $k$ and $\theta$. Pick any $a \in F(\theta)$. Consider $m \in M$ such that $m_{i}=(\theta, a, a, a, 0)$ for all $i \in N$. Then $l\left[S, \epsilon_{k}, m\right]=a$. We argue that $m \in N E\left(\Gamma_{O\left(S, \epsilon_{k}\right)}, \theta\right)$. Suppose player $i$ deviates from $m_{i}$ to $m_{i}^{\prime}=\left(\theta_{i}, a_{i}^{1}, a_{i}^{2}, a_{i}^{3}, z_{i}\right)$.

First, suppose $i \in S$. Then rule (ii) will be used when $N$ is the senate. In that case, $a$ is implemented if $l_{k}[i] \succ_{i}^{\theta} a$ and $a_{i}^{2}$ is implemented if $a \succeq_{i}^{\theta} l_{k}[i]$. On the other hand, rule (ii') will be used when $S$ is the senate. In that case, $a$ is implemented if $l_{k}[i] \succ_{i}^{\theta} a$ and $a_{i}^{3}$ is implemented if $a \succeq_{i}^{\theta} l_{k}[i]$. Therefore, if $l_{k}[i] \succ_{i}^{\theta} a$, then $l\left[S, \epsilon_{k},\left(m_{i}^{\prime}, m_{-i}\right)\right]=a$ whereas if $a \succeq_{i}^{\theta} l_{k}[i]$, then $l\left[S, \epsilon_{k},\left(m_{i}^{\prime}, m_{-i}\right)\right]=l_{k}[i]$. Hence, in either case, player $i$ does not improve by her deviation.

Second, suppose $i \in N \backslash S$. Then rule (ii) will be used when $N$ is the senate. In that case, $a$ is implemented if $a_{i}^{2} \succ_{i}^{\theta} a$ and $a_{i}^{2}$ is implemented if $a \succeq_{i}^{\theta} a_{i}^{2}$. On the other hand, $a$ will be implemented when $S$ is the senate. Therefore, if $a_{i}^{2} \succ_{i}^{\theta} a$, then $l\left[S, \epsilon_{k},\left(m_{i}^{\prime}, m_{-i}\right)\right]=a$ whereas if $a \succeq_{i}^{\theta} a_{i}^{2}$, then $l\left[S, \epsilon_{k},\left(m_{i}^{\prime}, m_{-i}\right)\right]$ is the lottery that assigns probability $\epsilon_{k}$ to $a_{i}^{2}$ and probability $1-\epsilon_{k}$ to $a$. Since preferences over lotteries are monotone, player $i$ does not improve by her deviation.

Therefore, $m \in N E\left(\Gamma_{O\left(S, \epsilon_{k}\right)}, \theta\right)$. 
Step 2. For all $k$ and $\theta \in \Theta,\left\{l\left[S, \epsilon_{k}, m\right]: m \in N E\left(\Gamma_{O\left(S, \epsilon_{k}\right)}, \theta\right)\right\} \subseteq F(\theta)$.

Fix $k$ and $\theta$. Pick any $\hat{m} \in N E\left(\Gamma_{O\left(S, \epsilon_{k}\right)}, \theta\right)$. For any $i$, let $\hat{m}_{i}=\left(\hat{\theta}_{i}, \hat{a}_{i}^{1}, \hat{a}_{i}^{2}, \hat{z}_{i}\right)$. We show that $l\left[S, \epsilon_{k}, \hat{m}\right] \in F(\theta)$.

First, suppose $\hat{m}$ is such that $g^{N}(\hat{m})$ follows rule (iii). This means for any $i \in N, g^{N}(\hat{m})$ is a most-preferred alternative in state $\theta$. If not, then $i$ can deviate to $m_{i}$ that differs from $\hat{m}_{i}$ only in the second and last components, with $a_{i}^{1}$ being one of her most-preferred alternatives in state $\theta$ and $z_{i}>\max _{j \neq i} \hat{z}_{j}$. If $N$ is the selected senate, then $g^{N}\left(m_{i}, \hat{m}_{-i}\right)$ follows rule (iii) and hence, $g^{N}\left(m_{i}, \hat{m}_{-i}\right)=a_{i}^{1}$. On the other hand, if $S$ is the selected senate, then one of the following in true:

- $i \in N \backslash S$ and $g^{S}\left(\left(m_{i}, \hat{m}_{-i}\right)^{S}\right)=g^{S}\left(\hat{m}^{S}\right)$.

- $i \in S$ and $g^{S}\left(\left(m_{i}, \hat{m}_{-i}\right)^{S}\right)$ follows rule (iii'). Then $g^{S}\left(\left(m_{i}, \hat{m}_{-i}\right)^{S}\right)=a_{i}^{1}$.

- $i \in S$ and $g^{S}\left(\left(m_{i}, \hat{m}_{-i}\right)^{S}\right)$ follows rule (ii'). There are two possibilities: $g^{S}\left(\hat{m}^{S}\right)$ followed either rule (i') or (ii'). However, in either case $g^{S}\left(\left(m_{i}, \hat{m}_{-i}\right)^{S}\right)=g^{S}\left(\hat{m}^{S}\right)$ since the alternative implemented in rule (ii') does not depend on the second and last components of player $i$ 's message.

Since preferences over lotteries are monotone, player $i$ will be better-off after the deviation to $m_{i}$, a contradiction. But if $g^{N}(\hat{m})$ is the most-preferred alternative in state $\theta$ for all players, then that contradicts the fact that $\mathcal{E}$ is an economic environment.

Second, suppose $\hat{m}$ is such that $g^{N}(\hat{m})$ follows rule (ii). Using a similar argument as in the previous case, $g^{N}(\hat{m})$ must be a most-preferred alternative in state $\theta$ for at least $n-1$ players, which again contradicts with $\mathcal{E}$ being economic.

Therefore, $g^{N}(\hat{m})$ follows rule (i). Hence, $\hat{m}_{i}=\left(\hat{\theta}, \hat{a}_{i}^{1}, \hat{a}, \hat{a}, 0\right)$ for all $i \in N$, where $\hat{a} \in F(\hat{\theta})$. If $\hat{a} \in F(\theta)$, then we are done. On the other hand, suppose $\hat{a} \notin F(\theta)$. Then, due to weak $S$-monotonicity of $F$, at least one of the following is true:

- There exist player $i_{k} \in N \backslash S$ and alternative $a_{k}^{\prime}$ such that $\hat{a} \succeq_{i_{k}}^{\hat{\theta}} a_{k}^{\prime}$ and $a_{k}^{\prime} \succ_{i_{k}}^{\theta} \hat{a}$. Then, thanks to rule (ii), player $i_{k}$ has an incentive to deviate to $\left(\hat{\theta}, \hat{a}_{i}^{1}, a_{k}^{\prime}, \hat{a}, 0\right)$.

- There exist player $i_{k} \in S$, lottery $l_{k}$, and alternatives $\hat{a}_{k}$ and $\tilde{a}_{k}$ such that

$$
l_{k}\left(\hat{a}_{k}\right)=\epsilon_{k}, \quad l_{k}\left(\tilde{a}_{k}\right)=1-\epsilon_{k}, \quad \hat{a} \succeq_{i_{k}}^{\hat{\theta}} l_{k} \text { and } l_{k} \succ_{i_{k}}^{\theta} \hat{a} .
$$

Thanks to rules (ii) and (ii'), player $i_{k}$ has an incentive to deviate to $\left(\hat{\theta}, \hat{a}_{i}^{1}, \hat{a}_{k}, \tilde{a}_{k}, 0\right)$.

Proof of Theorem 4.8: For any $\epsilon \in(0,1)$, define $\Gamma_{O(S, \epsilon)}=\left(\left(M_{i}\right)_{i \in N}, O(S, \epsilon),\left(g^{S^{\prime}}\right)_{S^{\prime} \in \mathcal{N}}\right)$ such that for all $i \in N$,

$$
M_{i}=\Theta \times A \times A \times \mathbb{Z}_{+} .
$$


Let a typical message $m_{i}$ be of the form $\left(\theta_{i}, a_{i}^{1}, a_{i}^{2}, z_{i}\right)$. For all $S^{\prime} \in\{N, S\}$, the outcome function $g^{S^{\prime}}$ is as follows:

(i) If for every player $i \in S^{\prime}, m_{i}=\left(\theta, a_{i}^{1}, a, 0\right)$ and $a \in F(\theta)$, then $g^{S^{\prime}}\left(\left(m_{i}\right)_{i \in S^{\prime}}\right)=a$.

(ii) If for $\left|S^{\prime}\right|-1$ players $i \neq j$ in $S^{\prime}, m_{i}=\left(\theta, a_{i}^{1}, a, 0\right)$ and $a \in F(\theta)$, but $m_{j}=$ $\left(\theta_{j}, a_{j}^{1}, a_{j}^{2}, z_{j}\right) \neq\left(\theta, a_{j}^{1}, a, 0\right)$, then

$$
g^{S^{\prime}}\left(\left(m_{i}\right)_{i \in S^{\prime}}\right)=\left\{\begin{array}{cl}
a & \text { if } a_{j}^{2} \succ_{j}^{\theta} a . \\
a_{j}^{2} & \text { if } a \succeq_{j}^{\theta} a_{j}^{2} .
\end{array}\right.
$$

(iii) In all other cases, $g^{S^{\prime}}\left(\left(m_{i}\right)_{i \in S^{\prime}}\right)=a_{j}^{1}$ where $j \in S^{\prime}$ is the player with the lowest index among those players in $S^{\prime}$ who announce the highest integer in the profile $\left(m_{i}\right)_{i \in S^{\prime}}$.

Finally, for all $S^{\prime} \in \mathcal{N} \backslash\{N, S\}$, the outcome function $g^{S^{\prime}}$ can be arbitrary.

Step 1. For all $\epsilon \in(0,1)$ and $\theta \in \Theta, F(\theta) \subseteq\left\{l[S, \epsilon, m]: m \in N E\left(\Gamma_{O(S, \epsilon)}, \theta\right)\right\}$.

Fix $\epsilon \in(0,1)$ and $\theta$. Pick any $a \in F(\theta)$. Consider $m \in M$ such that $m_{i}=(\theta, a, a, 0)$ for all $i \in N$. Then $l[S, \epsilon, m]=a$. We argue that $m \in N E\left(\Gamma_{O(S, \epsilon)}, \theta\right)$. Suppose player $i$ deviates from $m_{i}$ to $m_{i}^{\prime}=\left(\theta_{i}, a_{i}^{1}, a_{i}^{2}, z_{i}\right)$.

First, suppose $i \in S$. Then rule (ii) will be used irrespective of whether $N$ or $S$ is the senate. In either case, $a_{i}^{2}$ is implemented if $a \succeq_{i}^{\theta} a_{i}^{2}$ whereas $a$ is implemented if $a_{i}^{2} \succ_{i}^{\theta} a$. Thus, player $i$ does not improve by her deviation.

Second, suppose $i \in N \backslash S$. Then rule (ii) will be used when $N$ is the senate. In that case, $a_{i}^{2}$ is implemented if $a \succeq_{i}^{\theta} a_{i}^{2}$ whereas $a$ is implemented if $a_{i}^{2} \succ_{i}^{\theta} a$. On the other hand, $a$ is implemented when $S$ is the senate. Since the preferences over lotteries are monotone, player $i$ does not improve by her deviation.

Therefore, $m \in N E\left(\Gamma_{O(S, \epsilon)}, \theta\right)$.

Step 2. For all $\epsilon \in(0,1)$ and $\theta \in \Theta,\left\{l[S, \epsilon, m]: m \in N E\left(\Gamma_{O(S, \epsilon)}, \theta\right)\right\} \subseteq F(\theta)$.

Fix $\epsilon \in(0,1)$ and $\theta$. Pick any $\hat{m} \in N E\left(\Gamma_{O(S, \epsilon)}, \theta\right)$. For any $i$, let $\hat{m}_{i}=\left(\hat{\theta}_{i}, \hat{a}_{i}^{1}, \hat{a}_{i}^{2}, \hat{z}_{i}\right)$. We show that $l[S, \epsilon, \hat{m}] \in F(\theta)$.

First, suppose $\hat{m}$ is such that $g^{N}(\hat{m})$ follows rule (iii). This means for any $i \in N, g^{N}(\hat{m})$ is a most-preferred alternative in state $\theta$. If not, then $i$ can deviate to $m_{i}$ that differs from $\hat{m}_{i}$ only in the second and last components, with $a_{i}^{1}$ being one of her most-preferred alternatives in state $\theta$ and $z_{i}>\max _{j \neq i} \hat{z}_{j}$. If $N$ is the selected senate, then $g^{N}\left(m_{i}, \hat{m}_{-i}\right)$ follows rule (iii) and hence, $g^{N}\left(m_{i}, \hat{m}_{-i}\right)=a_{i}^{1}$. On the other hand, if $S$ is the selected senate, then one of the following in true:

- $i \in N \backslash S$ and $g^{S}\left(\left(m_{i}, \hat{m}_{-i}\right)^{S}\right)=g^{S}\left(\hat{m}^{S}\right)$. 
- $i \in S$ and $g^{S}\left(\left(m_{i}, \hat{m}_{-i}\right)^{S}\right)$ follows rule (iii). Then $g^{S}\left(\left(m_{i}, \hat{m}_{-i}\right)^{S}\right)=a_{i}^{1}$.

- $i \in S$ and $g^{S}\left(\left(m_{i}, \hat{m}_{-i}\right)^{S}\right)$ follows rule (ii). Then $g^{S}\left(\left(m_{i}, \hat{m}_{-i}\right)^{S}\right)=g^{S}\left(\hat{m}^{S}\right)$.

Since preferences over lotteries are monotone, player $i$ will be better-off after the deviation to $m_{i}$, a contradiction. But $g^{N}(\hat{m})$ being the most-preferred alternative in state $\theta$ for all players contradicts DTA-S.

Second, suppose $\hat{m}$ is such that $g^{N}(\hat{m})$ follows rule (ii). It must be that for $n-1$ players $i \neq j$ in $N, \hat{m}_{i}=\left(\hat{\theta}, \hat{a}_{i}^{1}, \hat{a}, 0\right)$ and $\hat{a} \in F(\hat{\theta})$, but $\hat{m}_{j}=\left(\hat{\theta}_{j}, \hat{a}_{j}^{1}, \hat{a}_{j}^{2}, \hat{z}_{j}\right) \neq\left(\hat{\theta}, \hat{a}_{j}^{1}, \hat{a}, 0\right)$. Using a similar argument as in the previous case, $g^{N}(\hat{m})$ must be a most-preferred alternative in state $\theta$ for all $i \neq j$. Since $F$ satisfies no-veto power, we have $g^{N}(\hat{m}) \in F(\theta)$. If $j \in N \backslash S$, then that contradicts DTA-S. Hence, $j \in S$. It follows from rule (ii) that $g^{S}\left(\hat{m}^{S}\right)=g^{N}(\hat{m}) \in F(\theta)$.

Finally, suppose $g^{N}(\hat{m})$ follows rule $(\mathrm{i})$. Hence, each player $i$ sends the message $\hat{m}_{i}=$ $\left(\hat{\theta}, \hat{a}_{i}^{1}, \hat{a}, 0\right)$, where $\hat{a} \in F(\hat{\theta})$. If $\hat{a} \in F(\theta)$, then we are done. On the other hand, suppose $\hat{a} \notin F(\theta)$. Then, since $F$ is Maskin monotonic, there exist player $i \in N$ and alternative $a^{\prime}$ such that $\hat{a} \succeq_{i}^{\hat{\theta}} a^{\prime}$ and $a^{\prime} \succ_{i}^{\theta} \hat{a}$. Then, thanks to rule (ii), player $i$ has an incentive to deviate to $\left(\hat{\theta}, \hat{a}_{i}^{1}, a^{\prime}, 0\right)$.

Since the above argument was made for all $\epsilon \in(0,1)$, it follows that $F$ is implementable by $S$-oligarchic democracy.

Proof of Theorem 4.9: For any $\epsilon \in(0,1)$, define $\Gamma_{O(S, \epsilon)}=\left(\left(M_{i}\right)_{i \in N}, O(S, \epsilon),\left(g^{S^{\prime}}\right)_{S^{\prime} \in \mathcal{N}}\right)$ such that for all $i \in N$,

$$
M_{i}=\Theta \times A \times A \times \mathbb{Z}_{+}
$$

Let a typical message $m_{i}$ be of the form $\left(\theta_{i}, a_{i}^{1}, a_{i}^{2}, z_{i}\right)$. The outcome function $g^{N}$ is as follows:

(i) If for every player $i \in N, m_{i}=\left(\theta, a_{i}^{1}, a, 0\right)$ and $a \in F(\theta)$, then $g^{N}(m)=a$.

(ii) If for $n-1$ players $i \neq j$ in $N, m_{i}=\left(\theta, a_{i}^{1}, a, 0\right)$ and $a \in F(\theta)$, but $m_{j}=\left(\theta_{j}, a_{j}^{1}, a_{j}^{2}, z_{j}\right) \neq$ $\left(\theta, a_{j}^{1}, a, 0\right)$, then

$$
\begin{aligned}
& j \in N \backslash S \Longrightarrow g^{N}(m)=\left\{\begin{array}{cl}
a & \text { if } a_{j}^{2} \succ_{j}^{\theta} a . \\
a_{j}^{2} & \text { if } a \succeq_{j}^{\theta} a_{j}^{2} .
\end{array}\right. \\
& j \in S \Longrightarrow g^{N}(m)=a .
\end{aligned}
$$

(iii) In all other cases, $g^{N}(m)=a_{j}^{1}$ where $j \in N$ is the player with the lowest index among those who announce the highest integer.

The outcome function $g^{S}$ is as follows: 
(i') If for every player $i \in S, m_{i}=\left(\theta, a_{i}^{1}, a, 0\right)$ and $a \in F(\theta)$, then $g^{S}\left(\left(m_{i}\right)_{i \in S}\right)=a$.

(ii') If for $|S|-1$ players $i \neq j$ in $S, m_{i}=\left(\theta, a_{i}^{1}, a, 0\right)$ and $a \in F(\theta)$, but $m_{j}=\left(\theta_{j}, a_{j}^{1}, a_{j}^{2}, z_{j}\right) \neq$ $\left(\theta, a_{j}^{1}, a, 0\right)$, then

$$
g^{S}\left(\left(m_{i}\right)_{i \in S}\right)=\left\{\begin{array}{cl}
a & \text { if } a_{j}^{2} \succ_{j}^{\theta} a . \\
a_{j}^{2} & \text { if } a \succeq_{j}^{\theta} a_{j}^{2} .
\end{array}\right.
$$

(iii') In all other cases, $g^{S}\left(\left(m_{i}\right)_{i \in S}\right)=a_{j}^{1}$ where $j \in S$ is the player with the lowest index among those players in $S$ who announce the highest integer in the profile $\left(m_{i}\right)_{i \in S}$.

Finally, for all $S^{\prime} \in \mathcal{N} \backslash\{N, S\}$, the outcome function $g^{S^{\prime}}$ can be arbitrary.

Step 1. For all $\epsilon \in(0,1)$ and $\theta \in \Theta, F(\theta) \subseteq\left\{l[S, \epsilon, m]: m \in N E\left(\Gamma_{O(S, \epsilon)}, \theta\right)\right\}$.

Fix $\epsilon \in(0,1)$ and $\theta$. Pick any $a \in F(\theta)$. Consider $m \in M$ such that $m_{i}=(\theta, a, a, 0)$ for all $i \in N$. Then $l[S, \epsilon, m]=a$. We argue that $m \in N E\left(\Gamma_{O(S, \epsilon)}, \theta\right)$. Suppose player $i$ deviates from $m_{i}$ to $m_{i}^{\prime}=\left(\theta_{i}, a_{i}^{1}, a_{i}^{2}, z_{i}\right)$.

First, suppose $i \in S$. Then rule (ii) will be used when $N$ is the senate and hence, $a$ will be implemented. On the other hand, rule (ii') will be used when $S$ is the senate. In that case, $a_{i}^{2}$ is implemented if $a \succeq_{i}^{\theta} a_{i}^{2}$ whereas $a$ is implemented if $a_{i}^{2} \succ_{i}^{\theta} a$. Since the preferences over lotteries are monotone, player $i$ does not improve by her deviation.

Second, suppose $i \in N \backslash S$. Then rule (ii) will be used when $N$ is the senate. In that case, $a_{i}^{2}$ is implemented if $a \succeq_{i}^{\theta} a_{i}^{2}$ whereas $a$ is implemented if $a_{i}^{2} \succ_{i}^{\theta} a$. On the other hand, $a$ is implemented when $S$ is the senate. Since the preferences over lotteries are monotone, player $i$ does not improve by her deviation.

Therefore, $m \in N E\left(\Gamma_{O(S, \epsilon)}, \theta\right)$.

Step 2. For all $\epsilon \in(0,1)$ and $\theta \in \Theta,\left\{l[S, \epsilon, m]: m \in N E\left(\Gamma_{O(S, \epsilon)}, \theta\right)\right\} \subseteq F(\theta)$.

Fix $\epsilon \in(0,1)$ and $\theta$. Pick any $\hat{m} \in N E\left(\Gamma_{O(S, \epsilon)}, \theta\right)$. For any $i$, let $\hat{m}_{i}=\left(\hat{\theta}_{i}, \hat{a}_{i}^{1}, \hat{a}_{i}^{2}, \hat{z}_{i}\right)$. We show that $l[S, \epsilon, \hat{m}] \in F(\theta)$.

First, suppose $\hat{m}$ is such that $g^{N}(\hat{m})$ follows rule (iii). This means for any $i \in N, g^{N}(\hat{m})$ is a most-preferred alternative in state $\theta$. If not, then $i$ can deviate to $m_{i}$ that differs from $\hat{m}_{i}$ only in the second and last components, with $a_{i}^{1}$ being one of her most-preferred alternatives in state $\theta$ and $z_{i}>\max _{j \neq i} \hat{z}_{j}$. If $N$ is the selected senate, then $g^{N}\left(m_{i}, \hat{m}_{-i}\right)$ follows rule (iii) and hence, $g^{N}\left(m_{i}, \hat{m}_{-i}\right)=a_{i}^{1}$. On the other hand, if $S$ is the selected senate, then one of the following in true:

- $i \in N \backslash S$ and $g^{S}\left(\left(m_{i}, \hat{m}_{-i}\right)^{S}\right)=g^{S}\left(\hat{m}^{S}\right)$.

- $i \in S$ and $g^{S}\left(\left(m_{i}, \hat{m}_{-i}\right)^{S}\right)$ follows rule (iii'). Then $g^{S}\left(\left(m_{i}, \hat{m}_{-i}\right)^{S}\right)=a_{i}^{1}$.

- $i \in S$ and $g^{S}\left(\left(m_{i}, \hat{m}_{-i}\right)^{S}\right)$ follows rule (ii'). Then $g^{S}\left(\left(m_{i}, \hat{m}_{-i}\right)^{S}\right)=g^{S}\left(\hat{m}^{S}\right)$. 
Since preferences over lotteries are monotone, player $i$ will be better-off after the deviation to $m_{i}$, a contradiction. But $g^{N}(\hat{m})$ being the most-preferred alternative in state $\theta$ for all players contradicts DTA-S.

Second, suppose $\hat{m}$ is such that $g^{N}(\hat{m})$ follows rule (ii). It must be that for $n-1$ players $i \neq j$ in $N, \hat{m}_{i}=\left(\hat{\theta}, \hat{a}_{i}^{1}, \hat{a}, 0\right)$ and $\hat{a} \in F(\hat{\theta})$, but $\hat{m}_{j}=\left(\hat{\theta}_{j}, \hat{a}_{j}^{1}, \hat{a}_{j}^{2}, \hat{z}_{j}\right) \neq\left(\hat{\theta}, \hat{a}_{j}^{1}, \hat{a}, 0\right)$. Using a similar argument as in the previous case, $g^{N}(\hat{m})$ must be a most-preferred alternative in state $\theta$ for all $i \neq j$. If $j \in N \backslash S$, then that contradicts DTA-S. Hence, $j \in S$. It follows from rule (ii) that $g^{N}(\hat{m})=\hat{a}$. If $S$ is the senate, then rule (ii') will be used. We argue that $g^{S}\left(\hat{m}^{S}\right)=\hat{a}$. Suppose not; then due to rule (ii'), $g^{S}\left(\hat{m}^{S}\right)=\hat{a}_{j}^{2}$. This means for any $i \in S \backslash\{j\}, \hat{a}_{j}^{2}$ is a most-preferred alternative in state $\theta$. If not, then such an $i$ can deviate to $m_{i}$ that differs from $\hat{m}_{i}$ only in the second and last components, with $a_{i}^{1}$ being one of her most-preferred alternatives in state $\theta$ and $z_{i}>\hat{z}_{j}$. If $N$ is the selected senate, then $g^{N}\left(m_{i}, \hat{m}_{-i}\right)$ follows rule (iii), whereas if $S$ is the selected senate, then $g^{S}\left(\left(m_{i}, \hat{m}_{-i}\right)^{S}\right)$ follows rule (iii'). Hence, $g^{N}\left(m_{i}, \hat{m}_{-i}\right)=g^{S}\left(\left(m_{i}, \hat{m}_{-i}\right)^{S}\right)=a_{i}^{1}$. Since preferences over lotteries are monotone, player $i$ will be better-off after the deviation to $m_{i}$, a contradiction. Thus, both $\hat{a}_{j}^{2}$ and $\hat{a}$ are in $P_{1}(i, \theta)$ for all $i \in S \backslash\{j\}$. By assumption, the environment satisfies unique-top condition. Hence, it must be that $\hat{a}_{j}^{2}=\hat{a}$. Now, if $\hat{a} \in F(\theta)$, then we are done. On the other hand, $\hat{a} \in F(\hat{\theta}), \hat{a} \notin F(\theta)$, and Maskin monotonicity of $F$ imply that there exists a $i^{\prime} \in N$ and $a^{\prime} \in A$ such that $\hat{a} \succeq_{i^{\prime}}^{\hat{\theta}} a^{\prime}$ but $a^{\prime} \succ_{i^{\prime}}^{\theta} \hat{a}$. But $\hat{a} \in P_{1}(i, \theta)$ for all $i \neq j$. Hence, $i^{\prime}=j$. But then $\hat{m}$ cannot be a Nash equilibrium since due to rules (ii) and (ii'), player $j$ has an improving deviation $m_{j}=\left(\hat{\theta}_{j}, \hat{a}_{j}^{1}, a^{\prime}, \hat{z}_{j}\right)$, a contradiction.

Finally, suppose $g^{N}(\hat{m})$ follows rule (i). Hence, each player $i$ send the message $\hat{m}_{i}=$ $\left(\hat{\theta}, \hat{a}_{i}^{1}, \hat{a}, 0\right)$, where $\hat{a} \in F(\hat{\theta})$. If $\hat{a} \in F(\theta)$, then we are done. On the other hand, suppose $\hat{a} \notin F(\theta)$. Then, since $F$ is Maskin monotonic, there exist player $i \in N$ and alternative $a^{\prime}$ such that $\hat{a} \succeq_{i}^{\hat{\theta}} a^{\prime}$ and $a^{\prime} \succ_{i}^{\theta} \hat{a}$. Then, thanks to rules (ii) and (ii'), player $i$ has an incentive to deviate to $\left(\hat{\theta}, \hat{a}_{i}^{1}, a^{\prime}, 0\right)$.

Since the above argument was made for all $\epsilon \in(0,1)$, it follows that $F$ is implementable by $S$-oligarchic democracy.

Proof of Theorem 5.4: Consider the $R(\bar{n})$-sampling mechanism $\Gamma_{R(\bar{n})}$ defined in the sketch of the proof.

Step 1. For any $\theta \in \Theta, F(\theta) \subseteq\left\{l[R(\bar{n}), m]: m \in N E\left(\Gamma_{R(\bar{n})}, \theta\right)\right\}$.

Pick any $a \in F(\theta)$ and consider $m \in M$ such that $m_{i}=(\theta, a, a, 0)$ for all $i \in N$. Then $l[R(\bar{n}), m]=a$. We argue that $m \in N E\left(\Gamma_{R(\bar{n})}, \theta\right)$. Suppose player $i$ deviates from $m_{i}$ to $m_{i}^{\prime}=\left(\theta_{i}, a_{i}^{1}, a_{i}^{2}, z_{i}\right)$. Pick any $S \in \mathcal{N}_{\bar{n}}$. First, suppose $S \in \mathcal{N}_{\bar{n}} \backslash \mathcal{N}_{\bar{n}}(i)$. Then rule (i) will be used when $S$ is the senate and hence, $a$ will be implemented. Second, suppose $S \in \mathcal{N}_{\bar{n}}(i)$. Since $|S|=\bar{n} \geq 4$, rule (ii) will be used when $S$ is the senate. In that case, $a$ is implemented 
if $a_{i}^{2} \succ_{i}^{\theta} a$ or $S \notin \mathbb{S}(i)$ and $a_{i}^{2}$ is implemented if $a \succeq_{i}^{\theta} a_{i}^{2}$ and $S \in \mathbb{S}(i)$. Since preferences over lotteries are monotone, player $i$ does not improve by her deviation. So, $m \in N E\left(\Gamma_{R(\bar{n})}, \theta\right)$.

Step 2. For any $\theta \in \Theta,\left\{l[R(\bar{n}), m]: m \in N E\left(\Gamma_{R(\bar{n})}, \theta\right)\right\} \subseteq F(\theta)$.

We show that if $\hat{m} \in N E\left(\Gamma_{R(\bar{n})}, \theta\right)$, then $l[R(\bar{n}), \hat{m}] \in F(\theta)$. For any $i$, let $\hat{m}_{i}=$ $\left(\hat{\theta}_{i}, \hat{a}_{i}^{1}, \hat{a}_{i}^{2}, \hat{z}_{i}\right)$. Let $i_{1}$ be the player with the lowest index amongst the players who announce the highest integer in $\hat{m}$.

First, suppose $\hat{m}$ is such that there exist at least three players, $i_{2}, i_{3}$ and $i_{4}$ such that $\left(\hat{\theta}_{i_{2}}, \hat{a}_{i_{2}}^{2}, \hat{z}_{i_{2}}\right) \neq\left(\hat{\theta}_{i_{3}}, \hat{a}_{i_{3}}^{2}, \hat{z}_{i_{3}}\right) \neq\left(\hat{\theta}_{i_{4}}, \hat{a}_{i_{4}}^{2}, \hat{z}_{i_{4}}\right)$ - notice that the second components of these players' messages are not being considered. Clearly, there is at most one player in $\left\{i_{2}, i_{3}, i_{4}\right\}$, denoted by $i_{k}$, such that $\left(\hat{\theta}_{i_{1}}, \hat{a}_{i_{1}}^{2}, \hat{z}_{i_{1}}\right)=\left(\hat{\theta}_{i_{k}}, \hat{a}_{i_{k}}^{2}, \hat{z}_{i_{k}}\right)$. Consider any $j \in N$ and pick any $S(j) \in \mathcal{N}_{\bar{n}}(j)$ such that (a) if $i_{k}=i_{1}$, then $\left\{i_{2}, i_{3}, i_{4}\right\} \subset S(j)$ and (b) if $i_{k} \neq i_{1}$, then $\left\{i_{1}, i_{2}, i_{3}, i_{4}\right\} \backslash\left\{i_{k}\right\} \subset S(j)$ (this is possible since $\bar{n} \geq 4$ ). Now if $S(j)$ is selected as the senate, then since there are three players in $S(j)$ with different messages, rule (iii) will be used. Moreover, since player $i_{1} \in S(j)$, the alternative announced by player $i_{1}$ in the second component of her message, $\hat{a}_{i_{1}}^{1}$, will be implemented when $S(j)$ is selected. We claim that for any $j \in N, \hat{a}_{i_{1}}^{1}$ is a most-preferred alternative in state $\theta$. Suppose not and let $j \in N$ be such that $\hat{a}_{i_{1}}^{1} \notin P_{1}(j, \theta)$. Let player $j$ deviate to $m_{j}$ that differs from $\hat{m}_{j}$ only in the second and last components, with $a_{j}^{1}$ being one of her most-preferred alternatives in state $\theta$ and $z_{j}>\hat{z}_{i_{1}}$. If the selected senate is some $S^{\prime} \in \mathcal{N}_{\bar{n}}(j)$, then one of the following will hold:

- $g^{S^{\prime}}\left(\left(m_{j}, \hat{m}_{-j}\right)^{S^{\prime}}\right)$ follows rule (iii). Then $g^{S^{\prime}}\left(\left(m_{j}, \hat{m}_{-j}\right)^{S^{\prime}}\right)=a_{j}^{1}$. Moreover, this will be the case when $S^{\prime}=S(j)$.

- $g^{S^{\prime}}\left(\left(m_{j}, \hat{m}_{-j}\right)^{S^{\prime}}\right)$ follows rule (ii). There are two possibilities: $g^{S^{\prime}}\left(\hat{m}^{S^{\prime}}\right)$ followed either rule (i) or (ii). However, in either case $g^{S^{\prime}}\left(\left(m_{j}, \hat{m}_{-j}\right)^{S^{\prime}}\right)=g^{S^{\prime}}\left(\hat{m}^{S^{\prime}}\right)$ since the alternative implemented in rule (ii) does not depend on the second and last components of player $j$ 's message.

On the other hand, if the selected senate is $S^{\prime} \in \mathcal{N}_{\bar{n}} \backslash \mathcal{N}_{\bar{n}}(j)$, then $g^{S^{\prime}}\left(\left(m_{j}, \hat{m}_{-j}\right)^{S^{\prime}}\right)=g^{S^{\prime}}\left(\hat{m}^{S^{\prime}}\right)$. Since preferences over lotteries are monotone, player $j$ will be better-off after the deviation to $m_{j}$, a contradiction. But if $\hat{a}_{i_{1}}^{1}$ is the most-preferred alternative in state $\theta$ for all players, then that contradicts $\mathcal{E}$ satisfying DTA- $N$.

Second, suppose $\hat{m}$ is such that there exist at least two players, $i_{2}$ and $i_{3}$ such that $\left(\hat{\theta}_{i_{2}}, \hat{a}_{i_{2}}^{2}, \hat{z}_{i_{2}}\right) \neq\left(\hat{\theta}_{i_{3}}, \hat{a}_{i_{3}}^{2}, \hat{z}_{i_{3}}\right)$ - again, notice that the second components of these players' messages are not being considered. If there exists a player $j$ such that $\left(\hat{\theta}_{i_{2}}, \hat{a}_{i_{2}}^{2}, \hat{z}_{i_{2}}\right) \neq$ $\left(\hat{\theta}_{i_{3}}, \hat{a}_{i_{3}}^{2}, \hat{z}_{i_{3}}\right) \neq\left(\hat{\theta}_{j}, \hat{a}_{j}^{2}, \hat{z}_{j}\right)$, then we are back in the first case. Hence, for every player $j$ there exists a player in $\left\{i_{2}, i_{3}\right\}$, denoted by $i(j)$, such that $\left(\hat{\theta}_{j}, \hat{a}_{j}^{2}, \hat{z}_{j}\right)=\left(\hat{\theta}_{i(j)}, \hat{a}_{i(j)}^{2}, \hat{z}_{i(j)}\right)$. Let $J_{2}=\left\{j \in N: i(j)=i_{2}\right\}$ and $J_{3}=\left\{j \in N: i(j)=i_{3}\right\}$. As before, $i_{1}$ is the player with the 
lowest index amongst the players who announce the highest integer in $\hat{m}$. Without loss of generality, suppose $i_{1} \in J_{2}$.

- Suppose there exists $j_{2} \neq i_{1}$ and $j_{3} \neq i_{3}$ such that $j_{2} \in J_{2}$ and $j_{3} \in J_{3}$. Consider any $j \in N$ and define $S(j)$ as follows. If $j \in\left\{i_{1}, j_{2}, i_{3}, j_{3}\right\}$, then let $S(j)$ be any set in $\mathcal{N}_{\bar{n}}(j)$ such that $\left\{i_{1}, j_{2}, i_{3}, j_{3}\right\} \subseteq S(j)$. If $j \in J_{2} \backslash\left\{i_{1}, j_{2}\right\}$, then let $S(j)$ be any set in $\mathcal{N}_{\bar{n}}(j)$ such that $\left\{i_{1}, j, i_{3}, j_{3}\right\} \subseteq S(j)$. Finally, if $j \in J_{3} \backslash\left\{i_{3}, j_{3}\right\}$, then let $S(j)$ be any set in $\mathcal{N}_{\bar{n}}(j)$ such that $\left\{i_{1}, j_{2}, i_{3}, j\right\} \subseteq S(j)$. In defining $S(j)$, we have made sure that there are at least two players each from $J_{2}$ and $J_{3}$, which is possible since $\bar{n} \geq 4$. Therefore, if $S(j)$ is selected as the senate, then rule (iii) will be used and hence, the alternative announced by player $i_{1}$ in the second component of her message, $\hat{a}_{i_{1}}^{1}$, will be implemented. As before, we can show that for any $j \in N, \hat{a}_{i_{1}}^{1}$ is a most-preferred alternative in state $\theta$, which contradicts $\mathcal{E}$ satisfying DTA- $N$.

- Suppose there exists $j_{2} \neq i_{1}$ such that $j_{2} \in J_{2}$ but $J_{3}=\left\{i_{3}\right\}$. Consider any $j \in N$ and let $S(j)$ be any set in $\mathcal{N}_{\bar{n}}(j)$ such that $\left\{i_{1}, j_{2}, i_{3}\right\} \subset S(j)$. Again, this is possible since $\bar{n} \geq 4$. If $S(j)$ is selected as the senate, then player $i_{3}$ is the only player in $S(j)$ who "disagrees". If $\hat{z}_{i_{1}} \neq 0$ or $\hat{a}_{i_{1}}^{2} \notin F\left(\hat{\theta}_{i_{1}}\right)$, then rule (iii) will be used in $S(j)$. In this case, $\hat{a}_{i_{1}}^{1}$ will be implemented in $S(j)$. As before, we can show that for any $j \in N$, $\hat{a}_{i_{1}}^{1}$ is a most-preferred alternative in state $\theta$, which contradicts $\mathcal{E}$ satisfying DTA- $N$.

On the other hand, if $\hat{z}_{i_{1}}=0$ and $\hat{a}_{i_{1}}^{2} \in F\left(\hat{\theta}_{i_{1}}\right)$, then rule (ii) will be used in $S(j)$. In fact, in this case, rule (ii) will be used for any $S \in \mathcal{N}_{\bar{n}}\left(i_{3}\right)$ (since $i_{3}$ is the only player in $S$ who "disagrees") whereas rule (i) will be used for any $S \in \mathcal{N}_{\bar{n}} \backslash \mathcal{N}_{\bar{n}}\left(i_{3}\right)$ (since all players in $S$ "agree"). Therefore, alternative $\hat{a}_{i_{1}}^{2}$ will be implemented for all $S \in \mathcal{N}_{\bar{n}} \backslash \mathbb{S}\left(i_{3}\right)$.

- Suppose $\hat{a}_{i_{3}}^{2} \succ_{i_{3}}^{\hat{\theta}_{i_{1}}} \hat{a}_{i_{1}}^{2}$. Then $\hat{a}_{i_{1}}^{2}$ is also implemented for all $S \in \mathbb{S}\left(i_{3}\right)$. If $\hat{a}_{i_{1}}^{2} \in F(\theta)$, then we are done. If $\hat{a}_{i_{1}}^{2} \notin F(\theta)$, then by Maskin monotonicity, there exists a player $i$ and $a^{\prime}$ such that $\hat{a}_{i_{1}}^{2} \succeq_{i}^{\hat{\theta}_{i_{1}}} a^{\prime}$ but $a^{\prime} \succ_{i}^{\theta} \hat{a}_{i_{1}}^{2}$. Let player $i$ deviate to $m_{i}^{\prime}=\left(\hat{\theta}_{i}, a^{\prime}, a^{\prime}, z^{\prime}\right)$, where $z^{\prime}>0=\hat{z}_{i_{1}} \geq \hat{z}_{i_{3}}$. If the selected senate is some $S^{\prime} \in \mathcal{N}_{\bar{n}}(i)$, then one of the following will hold:

* $g^{S^{\prime}}\left(\left(m_{i}^{\prime}, \hat{m}_{-i}\right)^{S^{\prime}}\right)$ follows rule (iii). Then $g^{S^{\prime}}\left(\left(m_{i}^{\prime}, \hat{m}_{-i}\right)^{S^{\prime}}\right)=a^{\prime}$.

* $g^{S^{\prime}}\left(\left(m_{i}^{\prime}, \hat{m}_{-i}\right)^{S^{\prime}}\right)$ follows rule (ii). Then $g^{S^{\prime}}\left(\left(m_{i}^{\prime}, \hat{m}_{-i}\right)^{S^{\prime}}\right)=a^{\prime}$ if $S^{\prime} \in \mathbb{S}(i)$ and $g^{S^{\prime}}\left(\left(m_{i}^{\prime}, \hat{m}_{-i}\right)^{S^{\prime}}\right)=g^{S^{\prime}}\left(\hat{m}^{S^{\prime}}\right)=\hat{a}_{i_{1}}^{2}$ if $S^{\prime} \notin \mathbb{S}(i)$.

Since there is a positive probability of selecting a senate in $\mathbb{S}(i)$, player $i$ has an incentive to deviate, which is a contradiction. 
- Suppose $\hat{a}_{i_{1}}^{2} \succeq_{i_{3}}^{\hat{\theta}_{i_{1}}} \hat{a}_{i_{3}}^{2}$. Then $\hat{a}_{i_{3}}^{2}$ is implemented for all $S \in \mathbb{S}\left(i_{3}\right)$. If $\hat{a}_{i_{1}}^{2}=\hat{a}_{i_{3}}^{2} \in$ $F(\theta)$, then we are done. If $\hat{a}_{i_{1}}^{2}=\hat{a}_{i_{3}}^{2} \notin F(\theta)$, then we are back in the previous case. So suppose $\hat{a}_{i_{1}}^{2} \neq \hat{a}_{i_{3}}^{2}$. We argue that $\left\{\hat{a}_{i_{1}}^{2}, \hat{a}_{i_{3}}^{2}\right\} \in P_{1}(j, \theta)$ for all $j \neq i_{3}$. If not, then let player $j$ deviate to $m_{j}$ such that $m_{j}$ differs from $\hat{m}_{j}$ only in the second and last components, with $a_{j}^{1}$ being her most-preferred alternative in state $\theta$ and $z_{j}>0=\hat{z}_{i_{1}} \geq \hat{z}_{i_{3}}$. If the selected senate is some $S^{\prime} \in \mathcal{N}_{\bar{n}}(j)$, then one of the following will hold:

* $g^{S^{\prime}}\left(\left(m_{j}, \hat{m}_{-j}\right)^{S^{\prime}}\right)$ follows rule (iii). Then $g^{S^{\prime}}\left(\left(m_{j}, \hat{m}_{-j}\right)^{S^{\prime}}\right)=a_{j}^{1}$. This will be the case for all $S^{\prime} \in \mathcal{N}_{\bar{n}}(j)$ such that $i_{3} \in S^{\prime}$. Furthermore, there exist $S_{1}^{\prime} \in \mathbb{S}\left(i_{3}\right)$ and $S_{2}^{\prime} \in \mathcal{N}_{\bar{n}} \backslash \mathbb{S}\left(i_{3}\right)$ such that $\left\{i_{3}, j\right\} \in S_{1}^{\prime}$ and $\left\{i_{3}, j\right\} \in S_{2}^{\prime}$. This is because $n>\bar{n} \geq 4$. Before the deviation, $g^{S_{1}^{\prime}}\left(\hat{m}^{S_{1}^{\prime}}\right)=\hat{a}_{i_{3}}^{2}$ while $g^{S_{2}^{\prime}}\left(\hat{m}^{S_{2}^{\prime}}\right)=\hat{a}_{i_{1}}^{2}$ with at least one alternative out of those two being strictly worse for $j$ than $a_{j}^{1}$. On the other hand, after the deviation, player $j$ will be able to implement her most-preferred alternative both when the senate is $S_{1}^{\prime}$ and $S_{2}^{\prime}$.

$* g^{S^{\prime}}\left(\left(m_{j}, \hat{m}_{-j}\right)^{S^{\prime}}\right)$ follows rule (ii). This will be the case for all $S^{\prime} \in \mathcal{N}_{\bar{n}}(j)$ such that $i_{3} \notin S^{\prime}$. Hence, $g^{S^{\prime}}\left(\hat{m}^{S^{\prime}}\right)$ followed rule (i). However, $g^{S^{\prime}}\left(\left(m_{j}, \hat{m}_{-j}\right)^{S^{\prime}}\right)=$ $g^{S^{\prime}}\left(\hat{m}^{S^{\prime}}\right)$ because the alternative implemented under rule (ii) does not depend on the second and last components of $j$ 's message.

On the other hand, if the selected senate is $S^{\prime} \in \mathcal{N}_{\bar{n}} \backslash \mathcal{N}_{\bar{n}}(j)$, then $g^{S^{\prime}}\left(\left(m_{j}, \hat{m}_{-j}\right)^{S^{\prime}}\right)=$ $g^{S^{\prime}}\left(\hat{m}^{S^{\prime}}\right)$. Since preferences over lotteries are monotone, player $j$ will be better-off after the deviation to $m_{j}$, a contradiction. But $\left\{\hat{a}_{i_{1}}^{2}, \hat{a}_{i_{3}}^{2}\right\} \in P_{1}(j, \theta)$ for all $j \neq i_{3}$ such that $\hat{a}_{i_{1}}^{2} \neq \hat{a}_{i_{3}}^{2}$ contradicts $\mathcal{E}$ satisfying top-coincidence condition.

- Suppose there exists $j_{3} \neq i_{3}$ such that $j_{3} \in J_{3}$ but $J_{2}=\left\{i_{1}\right\}$. This case can be argued like the previous case.

Therefore, $\hat{m}$ is such that $\left(\hat{\theta}_{i}, \hat{a}_{i}^{2}, \hat{z}_{i}\right)=(\hat{\theta}, \hat{a}, \hat{z})$ for all $i \in N$. Then $i_{1}=1$.

- Suppose either $\hat{z}>0$ or $\hat{a} \notin F(\hat{\theta})$. Consider any $j \in N$ and let $S(j)$ be any set in $\mathcal{N}_{\bar{n}}(j)$ such that $1 \in S(j)$. If $S(j)$ is selected as the senate, then rule (iii) will be used and hence, the alternative announced by player 1 in the second component of her message, $\hat{a}_{1}^{1}$, will be implemented. As before, we can show that for any $j \in N, \hat{a}_{1}^{1}$ is a most-preferred alternative in state $\theta$, which contradicts $\mathcal{E}$ satisfying DTA- $N$.

- Suppose $\hat{z}=0$ and $\hat{a} \in F(\hat{\theta})$. Then because of rule (i), for any $S \in \mathcal{N}_{\bar{n}}$, the alternative $\hat{a}$ is implemented when $S$ is the selected senate. If $\hat{a} \in F(\hat{\theta})$, then we are done. If $\hat{a} \notin F(\hat{\theta})$, then there exists a player $i$ and $a^{\prime}$ such that $\hat{a} \succeq_{i}^{\hat{\theta}} a^{\prime}$ but $a^{\prime} \succ_{i}^{\theta} \hat{a}$. Thanks to rule (ii), player $i$ has an incentive to deviate to $\left(\hat{\theta}, \hat{a}_{i}^{1}, a^{\prime}, 0\right)$. 


\section{References}

Benoît, J-P., Ok, E.A., 2008. Nash implementation without no-veto power. Games and Economic Behavior 64, 51-67.

Bergemann, D., Morris, S., 2008. Ex post implementation. Games and Economic Behavior $63,527-566$.

Bochet, O., 2007. Nash implementation with lottery mechanisms. Social Choice and Welfare $28,111-125$.

Danilov, V., 1992. Implementation via Nash equilibria. Econometrica 60, 43-56.

Jackson, M.O., 1992. Implementation in undominated strategies: A look at bounded mechanisms. Review of Economic Studies 59, 757-775.

Maskin, E., 1999. Nash equilibrium and welfare optimality. Review of Economic Studies 66, $23-38$.

Maskin, E., Sjöström, T., 2002. Implementation theory, in: Arrow, K.J., Sen, A.K., Suzumura, K., (Eds.), Handbook of Social Choice and Welfare 1. North-Holland, Amsterdam, pp 237288 .

McKelvey, R.D., 1989. Game forms for Nash implementation of general social choice correspondences. Social Choice and Welfare 6, 139-156.

Moore, J., Repullo, R., 1990. Nash implementation: A full characterization. Econometrica 58, 1083-1099.

Saijo, T., 1988. Strategy space reduction in Maskin's theorem: Sufficient conditions for Nash implementation. Econometrica 56, 693-700.

Serrano, R., 2004. The Theory of implementation of social choice rules. SIAM Review 46, 377-414.

Sjöström, T., 1991. On the necessary and sufficient conditions for Nash implementation. Social Choice and Welfare 8, 333-340.

Yamato, T., 1992. On Nash implementation of social choice correspondences. Games and Economic Behavior 4, 484-492. 